\title{
Proceedings of the Association of British Neurologists and Nederlandse Vereniging voor Neurologie, Aviemore, 4-6 May 1994
}

\section{Platform presentations}

CREUTZFELDT-JAKOB DISEASE: A COLLABORATIVE STUDY IN EUROPE DPWM Wientjens, RG Will, A Hofman. Erasmus University Medical School, Rotterdam, The Netherlands and the Western General Hospital, Edinburgh, UK

In various European countries there is anxiety about a possible link between animal spongioform encephalopathies and human encephalopathies, including Creutzfeldt-Jakob disease (CJD). The European collaborative study on CJD aims: (a) to study the frequency of human spongioform encephalopathies in Europe, in particular CJD, in relation to animal spongioform encephalopathies; and $(b)$ to assess the risk of CJD and other human spongioform encephalopathies in relation to genetic, occupational, and nutritional factors.

The European study on animal spongioform encephalopathies and CJD will comprise three parts: (a) the establishment of registries of CJD cases in European countries; $(b)$ a case-control study of risk factors for CJD on the basis of cases in the registries; (c) molecular genetic studies of CJD with material collected in the registries.

In a two year period 200 patients and 200 controls will be included in the collaborative case-control study. In the Dutch registry cases of CJD occurring in 1993 were ascertained. According to the diagnostic criteria for CJD there were 15 CJD cases in The Netherlands. Seven were classified as definite and eight probable between 1 January 1993 and 1 April 1994. The comparative incidence of CJD cases in France, Germany, Italy, The Netherlands and the United Kingdom is similar, despite greater variation in the incidence of bovine spongioform encephalopathy.

GENETIC HETEROGENEITY OF FAMILIAL HEMIPLEGIC MIGRAINE

MD Ferrari for Dutch Migraine Genetics Research Group. Leiden University Hospital, The Netherlands

Familial hemiplegic migraine (FHM) is a rare subtype of migraine with aura, which displays autosomal dominant inheritance. Identification of the FHM gene may help to unravel the pathogenesis of more common types of migraine. A linkage study has been started to identify the FHM gene.

Two unrelated multigenerational families displaying pure FHM with more than 240 microsatellite markers each were tested These and three additional families were tested specifically for linkage to chromosome $19 p$ markers.
Four of the five families had, as well as FHM, one or more family members with normal migraine with or without aura. Cosegregating symptoms were cerebellar ataxia in one and benign focal neonatal convulsions in another family. About $2800 \mathrm{cM}$ of the total genome in each of the two pedigrees was excluded in the random gene search. Linkage to markers for chromosome $19 \mathrm{p} 13$ locus was shown for three families; evidence against linkage was found in the two other families. Results of the two point and multipoint linkage analysis as well as the heterogeneity analysis will be discussed.

In some families, FHM is linked to chromosome $19 p 13$. Other gene loci also seem to be implicated. Clinical correlation to the linkage data and implications for the genetics of normal non-FHM migraine wil be discussed.

DOPPLER ULTRASOUND EVIDENCE OF EMBOLIC SOURCE

DG Grosset, D Georgiadis, A Kelman, I Bone, KR Lees. Western Infirmary and Institute of Neurological Sciences, Glasgow, UK

The detection of embolic materials with Doppler ultrasound, recently confirmed in experimental models, suggests a possible diagnostic role in patients at risk of embolism. Forty one patients with acute anterior circulation ischaemic stroke were studied within 48 hours of deficit; 27 patients with internal carotid artery (ICA) stenosis (21 symptomatic, studied 1-72 (median 4) months after deficit); and 30 healthy volunteers were also studied. In stroke cases, diagnostic subclassification was made from clinical, CT, and carotid and cardiac ultrasound data. In all cases, prolonged Doppler recordings were made from middle cerebral arteries (Nicolet TC2000, Warwick).

Emboli signals were detected in 29 of 41 cases of stroke $(71 \%), 24$ of 27 cases of ICA stenosis (89\%), and none of 30 controls. There was a correlation between emboli signals and the site and type of underlying disease. In patients with unilateral ICA stenosis the embolic count was significantly higher in the ipsilateral middle cerebral artery than contralaterally (14 $(10-22)$ v $2(0-7)$ signals per hour, median ( $95 \%$ confidence interval) $\mathrm{p}<0.0001)$. In patients with stroke, the emboli count was significantly higher in symptomatic than asymptomatic cerebral hemispheres ( $p<$ $0.05)$. No emboli were found in eight cases of lacunar stroke.

Intracranial Doppler emboli signals are common in ICA stenosis and in large vessel and cardioembolic stroke. Their absence in lacunar stroke is in keeping with a small vessel aetiology. The findings suggest that ongoing subclinical embolisation is clinically relevant and requires further study.

CEREBRAL HAEMODYNAMICS DURING GRAM NEGATIVE SEPTIC SHOCK: A TRANSCRANIAL DOPPLER STUDY

JS Straver, SJ Smith, GR de Ruiter, CJ Stam, RWM Keunen. Leyenburg Hospital, The Hague, The Netherlands

Dysfunction of the CNS usually occurs early in the course of sepsis and is associated with a significantly increased mortality. This study was designed to study the changes in cerebral haemodynamics during Gram negative septic shock (GNSS).

During seven days transcranial Doppler (TCD) studies of blood flow velocities (BFVs) in the middle cerebral (MCA) and internal carotid artery (ICA) were performed in patients with GNSS and with decreased consciousness in the intensive care unit. The TCD data were analysed in relation to several biochemical and haemodynamic indices as to a score of illness (APACHE-II-score).

Fourteen patients (10 men, four women, mean age 58.4 (SD 6.8) years) were examined. A severe GNSS existed in all patients based on systemic haemodynamic indices. Eight patients survived, six died. TCD showed increased MCA-BFV especially in days 3 to 6; ICA-BFV was in the normal range over the whole period. The MCA/ICA ratio was increased during the septic period. The mean MCA-BFV data correlated strongly with the APACHE-IIscore $\left(r_{\mathrm{s}}=0.81, \mathrm{p}=0.009\right)$.

In conclusion, a mild vasospasm of the basal cerebral arteries during these first seven days of sepsis could be demonstrated. Increased MCA-BFV is indicative for the severity of the disease. Non-survivors tended to have increased mean MCA-BFV but in this series did not reach significance.

RAPID HIGH RESOLUTION AND DYNAMIC "BLOOD FLOW" MAGNETIC RESONANCE IMAGING DETECTS SPINAL ARTERIOVENOUS MALFORMATIONS

JW Thorpe, BE Kendall, WI McDonald, DH Miller. Institute of Neurology, London, UK

Conventional MRI is relatively insensitive to spinal arteriovenous malformations (AVMs) and in particular dural fistulas (AVFs); diagnosis has required supine myelography followed by selective spinal angiography. Findings are reported using two recent advances: (a) rapid high resolution imaging with multiarray coils and fast 
spin echo; $(b)$ dynamic imaging during the passage of a gadolinium bolus. Six patients (five dural AVFs, one intramedullary AVM) and two controls have been studied. T1 and T2 weighted sagittal images of the whole cord were obtained with a multiarray receiver coil. Sagittal gradient echo images $\left(\mathrm{GE}_{24 / 25}\right.$, flip angle $\left.10^{\circ}\right)$ were then obtained at 3.5 second intervals after bolus injection of gadolinium-DTPA. Characteristic abnormalities were seen on high resolution images in all patients. In five, dynamic scanning showed transient signal reduction within the perimedullary venous plexus during bolus passage. The MRI findings correlated well with those from selective spinal angiography, the site of the arteriovenous shunt being localised to within one vertebral body in four patients.

With current MR imaging, myelography is probably unnecessary in the diagnosis of AVMs. Dynamic "blood flow" scanning is a useful adjunct to angiography as it may localise the site of an arteriovenous shunt.

LOSS OF CHROMOSOME 22 IN FAMILIAL ANAPLASTIC EPENDYMOMA

PCG Nijssen, RH Lekanne Deprez, CC Tijssen, A Hagemeijer, EPJ Arnoldus, JLJM Teepen, R Holl, MF Niermeijer. St Elisabeth Hospital, Tilburg, Erasmus University, Rotterdam, and University Hospital of Leiden, The Netherlands

Clinical features and genetic analysis of a family with anaplastic ependymomas are described.

Four affected members within a single generation of a family were investigated, and the data concerning their relatives in several generations were collected. Tumour histology and immunohistochemistry were obtained in three patients. Genotype analysis (fluorescent in situ hybridisation, restriction fragment length polymorphism analysis, and RNA-SSCP analysis of the NF2 gene) was performed on one of the tumours.

Two healthy brothers each had two affected sons. All four boys presented before five years of age with intraventricular tumours (three infratentorial, one supratentorial). Two boys died before the age of 3 . Neuroradiology, histology, and immunohistochemistry were similar in all tumours. Genetic analysis indicated monosomy of part of chromosome 22 in tumour cells. No mutations were found in the NF2 gene in tumour RNA.

In conclusion, a tumour suppressor gene on chromosome 22 may be involved in the pathogenesis of familial ependymal tumours.

ADVERSE EFFECTS OF RADIOTHERAPY IN LONG TERM LOW GRADE GLIOMA SURVIVORS MJB Taphoorn, JJ Heimans, A Klein Schiphorst, J Lindeboom, FJ Snoek, JG Wolbers, ABMF Karim, PC Huijgens. Free University Hospital, Amsterdam, The Netherlands

The role of early radiotherapy in the treatment of low grade gliomas (LGGs) is controversial. Retrospective analyses have shown a longer survival in patients with LGGs treated with radiotherapy, but radiotherapy may also negatively affect cognitive function and quality of life in long term survivors.
The impact of radiotherapy on cognitive function and quality of life was studied in 41 long term (mean 3.5 years) survivors with biopsy-proved LGG and without signs of tumour recurrence. Twenty patients had been treated with early radiotherapy; the other 21 patients only had surgery or biopsy. Nineteen patients with low grade haematological malignancies, surviving one to 15 years without CNS involvement, served as controls. Apart from the neurological and functional status, the patients' cognitive, affective and psychosocial status were also determined.

None of the LGG survivors had significant neurological impairment and their Karnofsky index was at least 70. More specific examination of cognitive functions, however (for example memory, word fluency, and concentration), and the affective status (Profile of Mood States) indicated that, compared with the control subjects, the patients with LGGs had significantly more cognitive disturbances and often showed fatigue and depressed moods. The two LGG groups, on the other hand, did not differ significantly for any of these indices.

In conclusion, radiotherapy had no deleterious impact on cognitive function or quality of life in patients treated for LGGs.

A GENETIC STUDY OF ATHETOID CEREBRAL PALSY

NA Fletcher, CD Marsden. Walton Centre for Neurology and Neurosurgery, Liverpool, and Institute of Neurology, London, UK

The cerebral palsies are a clinically and pathologically heterogenous group of conditions characterised by abnormal motor control and due to damage to the immature brain. Although traditionally attributed to perinatal hypoxic ischaemic encephalopathy, it is now recognised that in most cases, the aetiology is unknown. Genetic factors have not been thought to be important in cerebral palsy but previous studies have described affected relatives in a few cases and a paternal age effect has been described in athetoid cerebral palsy suggesting that some cases might arise by new dominant mutation.

In this study, we have looked at the question of genetic factors in athetoid cerebral palsy by means of a family study. The clinical features and family histories of 20 adults with athetoid cerebral palsy from 20 families have been analysed. Most of the patients had a progressive disorder which showed considerable clinical variability. In only three did the condition stabilise by 10 years of age and in seven there was deterio- ration after the age of 30 . Such late progression is difficult to explain in terms of a static perinatal lesion. Two patients developed a secondary cervical spondylotic myelopathy which mimicked progression of the cerebral disorder. Four patients had affected relatives and there were similar proportions of affected parents and sibs. The family data suggest genetic heterogeneity with autosomal recessive and dominant variants. The existence of an $\mathrm{X}$ linked form cannot be excluded and the demonstration of an increased paternal age effect among single cases suggests that some of these may arise because of fresh dominant genetic mutation.

MEASLES EPIDEMICITY AND SEASONALITY OF BIRTH IN MUTIPLE SCLEROSIS

PM Rothwell. Western General Hospital, Edinburgh, UK. (Introduced by CP Warlow)

To examine seasonality of birth in multiple sclerosis, the dates of birth (DOB) of 2356 patients with multiple sclerosis identified from death certificates between 1979 and 1991, and 11566 controls were obtained from the Registrar General for Scotland (RGS), and the DOB of 8160 patients discharged from hospital with a diagnosis of multiple sclerosis between 1968 and 1989 were obtained from the Scottish Hospitals Inpatient Survey (SHIPS). The years 1900-40 were divided into epidemic and non-epidemic years for measles, influenza, diphtheria, scarlet fever, whooping cough, and enteritis with mortality data from the RGS. Seasonality of multiple sclerosis births was examined in epidemic and nonepidemic years for each infection. Seasonality differed from controls only in years grouped according to measles epidemics (table, RGS cases $p<0.0001$, SHIPS cases $p<0.001$ ).

The excess of patients with multiple sclerosis compared with controls born during the second quarter peak of a measles epidemic, and the dearth of patients with multiple sclerosis in the third quarter, immediately after an epidemic, provide the first hard evidence in support of a link between early life viral infection and susceptibility to multiple sclerosis. See table below.

NEUROMYOTONIA WITH MYOCLONUS RESPONDING TO PLASMAPHERESIS

AR Wintzen, $M$ de Visser, JG van Dijk, BW Ongerboer de Visser. University Hospital Leiden, Amsterdam, The Netherlands

Two patients, a woman aged 43 (patient A), and a man aged 44 (patient B) presented with a five year history of limb muscle twitching. In addition patient $\mathbf{A}$ had action

\begin{tabular}{|c|c|c|c|c|}
\hline & \multicolumn{4}{|l|}{ Month of birth } \\
\hline & Fan-Mar & Apr-f̧un & ful-Sep & Oct-Dec \\
\hline \multicolumn{5}{|l|}{ RGS Cases } \\
\hline Epidemic years & 217 & 347 & 235 & 257 \\
\hline Non-epidemic years & 242 & 218 & 241 & 212 \\
\hline Odds ratio $(95 \% \mathrm{CI})$ & $0.90(0.73-1.09)$ & $1 \cdot 45(1 \cdot 19-1 \cdot 77)$ & $0.75(0.61-0.92)$ & $1.00(0.81-1.23)$ \\
\hline \multicolumn{5}{|l|}{ SHIPS Cases: } \\
\hline Epidemic years & 1132 & 1204 & 985 & 991 \\
\hline Non-epidemic years & 994 & 995 & 975 & 884 \\
\hline Odds ratio $(95 \% \mathrm{CI})$ & $1.02(0.93-1.13)$ & $1 \cdot 11(1 \cdot 01-1 \cdot 23)$ & $0.87(0.79-0.97)$ & $1.00(0 \cdot 90-1 \cdot 11)$ \\
\hline \multicolumn{5}{|l|}{ Controls: } \\
\hline Epidemic years & 1483 & 1533 & 1392 & 1365 \\
\hline Non-epidemic years & 1452 & 1491 & 1396 & 1444 \\
\hline Odds ratio $(95 \% \mathrm{CI})$ & $1.03(0.95-1.12)$ & $1.04(0.96-1 \cdot 13)$ & $1.00(0.92-1.09)$ & $0.94(0.86-1.02)$ \\
\hline
\end{tabular}


myoclonus of the proximal leg muscles, preventing her from standing on one leg or descending stairs. Patient $\mathbf{B}$ had similar myoclonus of one arm and one leg preventing him from adequately driving a car. In both patients myokymia-like spontaneous muscle activity was seen in many muscles during rest. Electromyography showed intermittent bursts of high frequency potentials, typical of neuromyotonia. In both patients spontaneous muscle activity and myoclonus were abolished by plasmapheresis.

It is concluded that incapacitating myoclonus may be part of the syndrome of acquired neuromyotonia, and responds to plasmapheresis.

A RANDOMISED DOUBLE BLIND CONTROILED STUDY OF INTRAVENOUS IMMUNOGLOBULIN IN THE LAMBERT-EATON MYASTHENIC SYNDROME

P Bain, G Elrington, E Goodger, S Misbah, P Panegyres, K MacPherson, $H$ Chapel, J Newsom-Davis. University of Oxford and Radcliffe Infirmary, UK

The Lambert-Eaton myasthenic syndrome (LEMS) is caused by IgG autoantibodies to nerve terminal voltage gated calcium channels. The efficacy of a two day course of intravenous immunoglobulin (IVIg; 1 $\mathrm{g} / \mathrm{kg} /$ day Gammagard) was studied in a double blind placebo controlled crossover trial. Ten patients (four men, six women; mean age 53.4 years) with LEMS and without detectable malignancies were treated. Mean duration of illness was $9 \cdot 8$ (range 4-21) years. Current treatments were continued during the trial. Nine patients completed the trial; five had headache and two had flu-like symptoms for 48 hours after IVIg. There was a subjective benefit after IVIg in eight patients. A statistically significant (Wilcoxon signed rank sum test, $p<$ 0.05 ) objective improvement in overall muscle strength, drinking time (for $150 \mathrm{ml}$ of water), and vital capacity was found two weeks after IVIg and this persisted for about six weeks. The peak effect, seen four weeks after IVIg had been given, produced a median improvement of about $18 \%$ in overall muscle strength $(p<0.05), 8 \%$ in vital capacity $(\mathrm{p}<0.01)$, and $35 \%$ in drinking time $(p<0.05)$ (expressed as percentages of the respective lower limits of normal). Placebo $(0.3 \%$ albumin $)$ had no significant effect.

In conclusion, IVIg may be a useful additional treatment for LEMS.

A DOUBLE BLIND PLACEBO CONTROLLED TRIAL OF INTRAVENOUS IMMUNOGLOBULIN AND FOLLOW UP STUDY IN MOTOR NEUROPATHY WITH CONDUCTION BLOCK AND/OR GMI ANTIBODIES

JHJ Wokke, LH van de Berg, H Kerkhoff, PL Oey, H Franssen, FGI Jennekens. University Hospital Utrecht, The Netherlands

Intravenous immunoglobulins (IVIgs) may be beneficial in patients with multifocal motor neuropathy (MMN) or motor neuron disease (MND) with conduction block (CB) and/or high serum GM1 antibody titres (hGM1). This possible effect has not been examined in a placebo-controlled study. Six patients were treated with IVIg
$(0.4 \mathrm{~g} / \mathrm{kg}$ for five days) in an open trial. Improvement was measured with myometry, the MRC grading system, and the Rankin scale. Three patients with $\mathrm{CB}$, one patient with CB and hGM1, and one with only hGM1 responded transiently to IVIg. Another patient with only hGM1 did not improve. The effect in one patient with CB lasted for two years. Next, the four patients with transient improvement were treated in a double blind placebo controlled crossover study during which they received randomly two IVIg and two placebo treatments.

The two patients with $\mathrm{CB}$ and the patient with hGM1 responded favourably to IVIg but not to placebo. One patient with CB and hGM1 did not respond. The effect was less strong and lasted for a short duration compared with other open studies. During a one year follow up period these four patients and another patient with $C B$ who did not participate in this study remained stable with single day treatment with IVIg $(0 \cdot 4 \mathrm{~g} /$ day $)$ every one to two weeks.

In conclusion, IVIg is effective in MMN, but responders should be selected in a placebo controlled trial.

ANTIBODIES TO GLYCOCONJUGATES IN PERIPHERAL NEUROPATHY

LH van den Berg, JHJ Wokke, IFAM Mollee, NC Notermans, PR Bär. University Hospital, Utrecht, The Netherlands

Antibodies to myelin associated glycoprotein (MAG), sulphatide, and the GM1 ganglioside were studied in patients with peripheral neuropathies. Antibodies to MAG were found in patients with a demyelinating sensorimotor polyneuropathy associated with $\operatorname{IgM}$ monoclonal gammopathy. Light microscopy of the sural nerve showed demyelination and IgM deposits and electronmicroscopy showed widening of myelin lamellae at the major dense line. Antibodies to the glycolipids, sulphatide, and the GM1 ganglioside were detected in serum of a large number of patients with neuropathies (135) as well as neurological (120), immunological (80), and normal (50) controls. Raised (higher than controls) sulphatide antibody titres were detected in six patients with neuropathy, who all had profound sensory loss. Two patients had a mild sensory and painful axonal neuropathy associated with an IgM monoclonal gammopathy. Three patients with increased titres of sulphatide antibodies also had MAG antibody activity and a demyelinating sensorimotor neuropathy. One patient had Guillain-Barré syndrome. This patient had the most profound sensory loss of all 22 patients with GBS who were tested for sulphatide antibodies. GM1 antibody titres were raised in patients with predominantly motor neuropathies. Raised titres were found in patients with Guillain-Barré syndrome, multifocal motor neuropathy, and lower motor neuron disease. Patients with Guillain-Barré syndrome and with raised GM1 antibodies had a worse prognosis than patients with Gullain-Barré syndrome but without GM1 antibodies.

PATHOPHYSIOLOGICAL EFFECTS OF ANTIBODIES IN MILLER FISHER SYNDROME M Roberts, $H$ Willison, A Vincent, J Newsom-Davis. Institute of Molecular Medicine, Oxford and University of Glasgow, UK
The Miller Fisher Syndrome (MFS) variant of Guillain-Barré syndrome is characterised by the presence of serum IgG anti-GQ1b ganglioside antibodies. Previous studies have shown that MFS sera can block neurotransmitter release from motor nerve terminals in the mouse phrenic nerve-diaphragm preparation. This occurs within four hours of application in vitro, preceded by an eightfold increase in miniature endplate potential (mepp) frequency. Control sera and a convalescent anti-GQ1b negative serum were without effect.

It has now been shown, in a further MFS case, that the activity is contained in the Ig fraction: plasma or a crude IgG preparation increased mepp frequency in the mouse diaphragm from the control value of $0.5 / \mathrm{s}$ to up to 6/s during the first two hours and caused block of neuromuscular transmission at 3.5 hours. In a patient with chronic paraproteinaemic neuropathy (CPN), with cold agglutinin IgM reacting with GQ1b, neither serum nor purified IgM affected mepp frequency after incubation at room temperature. An increased frequency of $2 \cdot 5 / \mathrm{s}$ was seen, however after three hours in purified IgM at $4^{\circ} \mathrm{C}$.

These findings suggest that the serum factor causing muscle paralysis in MFS is in the antibody fraction, and may be GQ1b ganglioside antibody.

TREATMENT OF THE GUILLAIN-BARRÉ SYNDROME WITH HIGH DOSE IMMUNE GLOBULINS COMBINED WITH

METHYLPREDNISOLONE: A PILOT STUDY LH Visser, PLM Schmitz, PA van Doorn, FGA van der Meché for the Dutch Guillain-Barré Study Group, University Hospital/Dijkzigt and Erasmus University, Rotterdam, The Netherlands

The effect of high dose immune globulins combined with methylprednisolone in patients with the Guillain-Barré syndrome was assessed.

In an open study 25 patients with Guillain-Barré syndrome were treated for five days with intravenous immune globulins in a dose of $0.4 \mathrm{~g}$ per kg bodyweight per day and $0.5 \mathrm{~g}$ methylprednisolone intravenously per day. The results of this combined treatment were compared with the results from a group of 74 patients who were treated with immune globulins only in a recent Dutch Guillain-Barré trial.

In the methylprednisolone-immune globulin group 19 of 25 patients (76\%) improved by one or more functional grades after four weeks, compared with 39 of 74 patients $(53 \%)$ treated with immune globulin alone $(p=0.04)$. Also the median time required to reach the stage of walking independently was reduced in the methylprednisolone-immune globulin treatment group.

This pilot study suggests that combined treatment with methylprednisolone and immune globulin in patients with the Guillain-Barré syndrome is more effective than treatment with immune globulin alone. A randomised clinical trial might confirm this.

EARLY ONSET FACIOSCAPULOHUMERAL MUSCULAR DYSTROPHY

OF Brouwer, GW Padberg, C Wijmenga, RR Frants. Leiden University and Nijmegen University, The Netherlands 
The occurrence and the clinical and genetic variability of early onset facioscapulohumeral muscular dystrophy (FSHD) has been determined.

A group of 96 patients from 17 families with autosomal dominant FSHD, nine sporadic cases of FSHD, and 35 children at risk for having the FSHD gene were searched for the presence of early onset cases. Criteria for early onset FSHD were (a) signs or symptoms of facial weakness before the age of 5 , and (b) signs or symptoms of shoulder girdle weakness before the age of 10 years.

Six early onset cases (four familial, two sporadic) were identified. Four out of five cases showed an abnormal DNA fragment with the probe p13E-11, which has been shown to detect DNA rearrangements in patients with FSHD. The clinical symptoms in these early onset cases showed great variability with respect to rate of progression of muscle weakness, the association of hearing loss and retinopathy, and the expression of the disorder in parents and siblings.

It has been suggested that infantile FSHD is a separate form of the disease, often accompanied by sensorineural hearing loss and retinal telangiectasia. These findings, as well as the recent discovery that both hearing loss and retinal vasculopathy are part of FSHD, suggest that early onset FSHD is not a separate entity, but part of a wide clinical spectrum of FSHD.

THE COURSE OF HEREDITARY MOTOR AND SENSORY NEUROPATHY TYPE I

JE Hoogendijk, $M$ de Visser, PA Bolhuis, AAM Hart, BW Ongerboer de Visser. Academic Medical Center, Amsterdam, The Netherlands

Hereditary motor and sensory neuropathy type I (HMSN I) is known as a degenerative disorder with a slowly progressive course. This cross sectional study was undertaken to reevaluate the clinical and neurographic course.

Forty four people, aged 8-68 (mean 34) years, from six families carrying the PMP22 duplication, were investigated to determine the Neurological Disability Score (NDS) and its motor (M), sensory (S), and reflexes (R) subsets for clinical severity, the median nerve motor conduction velocity (MNCV), and the distally evoked compound muscle action potential (CMAP) amplitude. Correlations were analysed by random effects analysis of covariance.

The results showed that age was significantly related only to S-NDS, and not to NDS, W-NDS, R-NDS, MNCV, or CMAP amplitude. NDS and its subsets were strongly inversely related to $\mathrm{MNCV}$, and, to a lesser extent, to CMAP amplitude.

These results suggest that the primary pathological process in HMSN I is not, or is only slightly, active after childhood. The clinical progression seen in occasional patients may be due to other factors.

GLYCATION OF PERIPHERAL NERVE PROTEINS IN DIABETES MELLITUS

C Ryle, M Donaghy. University of Oxford, UK

The biological mechanism(s) responsible for polyneuropathy in diabetes remain undetermined. Irreversible non-enzymatic glycation of proteins underlies some tissue complications of diabetes, and the vulnerability of neural tissues to this chemical modification has been investigated. Proteins from the axonal cytoskeleton and myelin fractions of peripheral nerve and spinal cord from diabetic rats have been assayed for the early glycation adduct (measured as furosine); the advanced glycation endproduct (AGE) pentosidine; and for protein cross linkage. In diabetic animals all these measures of glycation were higher in nerve than spinal cord; and nerve cytoskeleta AGE concentrations rose both with diabetes and with time. Autoradiography showed increased borotritride labelling of glycated neurofilament subunits in the cytoskeletal fractions of diabetic peripheral nerve. These results show that the peripheral nerve cytoskeletal fraction is vulnerable to nonenzymatic glycation, including AGE formation, in diabetic rats, and to a lesser extent in normal rats.

In human sural nerves obtained from diabetic and non-diabetic amputation specimens, the early reversible glycation adduct, measured as furosine, did not increase with age in either group, and did not differ significantly between diabetic patients and controls in either protein fraction. There was no age related accumulation of the irreversible $A G E$, pentosidine, over the range 42-87 years. Pentosidine levels per unit protein were significantly increased in diabetic patients relative to controls in both cytoskeletal (5.96 $v 4.47 ; \mathrm{p}=0.037)$ and myelin protein $(1.35 v 0.69 ; \mathrm{p}=0.023)$ fractions. These results show that increased AGE accumulation occurs in cytoskeletal, as well as myelin, peripheral nerve proteins in diabetic patients. This suggests a possible new mechanism contributing to the axonal degeneration polyneuropathy of diabetes based on irreversible glycation of axonal cytoskeletal proteins causing cross linkage and altered function.

PERIPHERAL NEUROPATHY INDUCED BY PACIITAXEL (TAXOL)

TJ Postma, JJ Heimans, JB Vermorken. Free University Hospital, Amsterdam, The Netherlands

The occurrence, severity, dose dependency, and reversibility of paclitaxel-induced neuropathy has been evaluated in patients treated with single agent paclitaxel at three different dosages $\left(135,175\right.$, and $\left.250-300 \mathrm{mg} / \mathrm{m}^{2}\right)$, given every three weeks by three hours intravenous infusion.

Twenty seven patients with breast cancer or ovarian cancer were prospectively evaluated neurologically before starting paclitaxel, after every other treatment course, and when off therapy, with a questionnaire and assessment of sensory and motor functions. Quantitatively, vibratory perception threshold (vibrameter) and grip strength (dynamometer) were measured.

In six patients, of whom four had cisplatin-induced neuropathy at baseline, treatment with $135 \mathrm{mg} / \mathrm{m}^{2}$ induced only minor neuropathic symptoms and signs. In 14 patients treated with $175 \mathrm{mg} / \mathrm{m}^{2}$, neuropathy was more pronounced and dose limiting in one. In seven patients treated with $250-300 \mathrm{mg} / \mathrm{m}^{2}$ neuropathy was significant and dose limiting in five. Paclitaxel induced a predominantly sensory neuropathy.
According to data from 10 patients who were three to four months off therapy, paclitaxel-induced neuropathy is, at least partly, reversible.

It is concluded that paclitaxel-induced neuropathy is dose dependent, occurring with higher cumulative dose and higher dose per course. Dose limiting neurotoxicity can be expected in patients treated with 250 $\mathrm{mg} / \mathrm{m}^{2}$ or more every three weeks.

AN EPIDEMIC OF OPTIC NEUROPATHY AND PAINFUL SENSORY NEUROPATHY IN CUBA PK Thomas, G Plant, PJ Baxter, R Santiago Luis. Royal Free Hospital School of Medicine and Institute of Neurology, London; Addenbrooke's Hospital, Cambridge, UK; and Instituto de Neurologia y Neurocirurgia, Havana, Cuba

In 1991 cases of bilateral optic neuropathy began to occur in western Cuba. Examination showed symmetric central or caecocentral scotomata, optic disc pallor, and loss of fibres in the maculopapillary bundle. Subsequently this came to be associated with painful burning paraesthesiae in the feet, mild weakness in the legs, and sometimes urinary precipitancy. Examination showed distal sensory loss in the limbs, brisk knee jerks, absent ankle jerks, and flexor plantar responses as well as bilateral optic neuropathy. Angular stomatitis was sometimes present. Nerve conduction studies indicated a predominantly sensory axonopathy, confirmed by sural nerve biopsy. The disorder spread to other regions of Cuba and by mid-1993, over 50000 cases had been notified. In the cases that occurred later in the epidemic, sensory symptoms predominated. After the administration of $\mathbf{B}$ vitamins to the whole island the epidemic subsided. The combination of bilateral optic neuropathy, painful sensory neuropathy, and angular stomatitis suggests that the disorder has affinities to Strachan's syndrome, originally described in the West Indies in the last century, and on various other occasions since. A basis in nutritional deficiency is assumed, possibly with a superimposed toxic component, but the precise aetiological factors have not been identified.

THE EPIDEMIOLOGY OF MOTOR NEURON DISEASE IN SCOTLAND

R Davenport, A Chancellor, C Warlow, RJ Swingler. University of Edinburgh and Dundee Royal Infirmary, UK

The Scottish Motor Neuron Disease (MND) Register is the largest prospective population based study of MND in the world. Five hundred and thirty five patients were registered between 1989 and 1993 and the incidence is $2 \cdot 1 / 10^{5} /$ year. Two hundred and ninety eight $(56 \%)$ were men $\left(2 \cdot 42 / 10^{5} /\right.$ year $)$ and $237(44 \%)$ were women $\left(1 \cdot 8 / 10^{5} /\right.$ year $)$. The mean age at registration is 64.8 years $(63.1$ for men and 66.8 for women). Two hundred and seventy two (51\%) patients were thought to have definite disease by local criteria and $263(49 \%)$ had probable or suspected MND. Three hundred and sixty seven $(69 \%)$ patients have died and mean survival is 24 months from onset and 12 months from diagnosis Old age and bulbar onset are associated with a worse prognosis. 
The geographic distribution was analysed in 367 patients and shows a significant inverse association with socioeconomic deprivation. A case-control study of 103 patients has shown no difference in social class in adult life or childhood and this pattern may be explained by underascertainment of older patients in less affluent areas. Cases were more likely to recall fractures before onset and occupational exposure to toxins but no single agent was implicated. Genetic factors are clearly implicated in the 25 patients $(4 \cdot 7 \%)$ with a family history of MND. Gompertzian analyses of agespecific incidence rates provide some evidence that sporadic MND also develops in a susceptible subgroup of the Scottish population.

CU-ZN SUPEROXIDE DISMUTASE (SOD1) MUTATIONS AND SPORADIC MOTOR NEURON DISEASE IN SCOTLAND

C Jones, D Brock, C Warlow, RJ Swingler. Universities of Edinburgh and Dundee, UK

$\mathrm{Cu}-\mathrm{Zn}$ superoxide dismutase gene (SOD1) mutations have been detected in 4/56 unrelated patients with sporadic MND drawn from the Scottish Motor Neuron Disease Register. Those with a family history (an affected relative) were excluded from the study. In one case an affected man was illegitimate but there was no family history of MND on the maternal side. In the other three cases there was no family history of MND in either paternal or maternal branches of the family.

In three of the patients an ATT-ACT nucleotide substitution was detected in codon 113 of exon 4 . This mis-sense mutation substitutes threonine for isoleucine and has been found in familial MND. A fourth patient had a novel glutamic acid to lysine mutation in codon 21 of exon 1 . No mutations were detected in other exons, or in samples taken from 80 controls. The findings indicate that germline mutations may be a more common cause of sporadic MND than hitherto suspected and this has important implications for the counselling of the immediate families. They also support the hypothesis that MND is associated with abnormalities of free radical metabolism and mutations in other candidate genes are now being sought.

DIFFERENTIAL DIAGNOSIS IN SPINOBULBAR MUSCULAR ATROPHY: CLINICAL AND MOLECULAR ASPECTS GJ Jöbsis, ES Louwerse, $M$ de Visser, JHTM Koelman, WM Wiersinga, HFM Busch, AO Brinkman, PA Bolhuis, F Baas, JMBV de Jong. Academic Medical Centre, Amsterdam, and Dijkzigt Ziekenhuis, Rotterdam, The Netherlands

The aim was firstly, to evaluate the clinical features of Kennedy's disease in comparison with those of other, atypical forms of spinobulbar muscular atrophy (SBMA), and secondly, to establish whether atypical SBMA is related to mutations in the androgen receptor gene.

$\mathrm{X}$ linked spinobulbar muscular atrophy (X-SBMA, Kennedy's disease) is a nosological entity based on clinical criteria and is caused by an enlarged trinucleotide repeat in the androgen receptor gene.
Atypical forms of SBMA exist, however, which share many characteristics with Kennedy's disease.

The clinical features of seven patients presenting with slowly progressive SBMA were evaluated and compared with those of three patients with Kennedy's disease. Also, the results of further investigations including endocrinological studies were evaluated. The androgen receptor gene was analysed for the length of the CAG repeat. The entire coding sequence was screened for mutations by means of single stranded conformation polymorphism (SSCP).

The age of onset, bulbar involvement, signs of reduced androgen sensitivity, cramps, fasciculations, tremor, plantar responses, benign course, EMG, SSEP, routine blood tests, and endocrinological tests did not differentiate Kennedy's disease from atypical SBMA. Site of onset, distribution of weakness, vibration sense, tendon reflexes of the lower limb, and pseudobulbar reflexes were helpful to distinguish atypical SBMA from Kennedy's disease. The androgen gene of the atypical SBMA patients had normal CAG repeat length and showed normal SSCP patterns, thus rendering any mutation within the coding sequence highly unlikely.

It is concluded that atypical SBMA is not caused by exonic mutations of the androgen receptor gene and can be differentiated from Kennedy's disease on clinical grounds.

RAISED SPINAL CONCENTRATIONS OF SELENIUM AND IRON AND GLUTATHIONE PEROXIDASE ACTIVITY IN SPORADIC MOTOR NEURON DISEASE

PJ Shaw, PG Ince, JM Candy, D Mantle, F McArthur, L Tandon, WD Ehmann, WR Markesbery. University of Newcastle upon Tyne, UK and the University of Kentucky, USA

Oxygen free radical damage has been implicated in the pathogenesis of familial motor neuron disease (MND) in patients with mutations of the $\mathrm{Cu} / \mathrm{Zn}$ superoxide dismutase (SOD1) gene. At present the relevance of this important finding to sporadic MND remains unknown. Certain metals and trace elements may be linked with free radical pathways in the CNS. Selenium (Se) is an essential trace element and over $40 \%$ of the $\mathrm{Se}$ in mammalian brain is contained in the Se dependent enzyme glutathione peroxidase. This enzyme acts in concert with SOD1 forming a major part of the oxygen free radical detoxifying mechanism in neurons. Iron, in its ferrous form, is also a potential source of free radicals in biological systems.

The aim of this study was to compare bulk tissue concentrations of 10 elements in spinal cord tissue from MND and control cases by neutron activation analysis. Uncontaminated L1 spinal cord samples were processed from 38 sporadic MND subjects and 22 controls.

There were significant increases in mean tissue concentrations of Se $(p<0.0001)$ and iron $(p<0.009)$ in the MND group. It is postulated that the increased Se in MND spinal cord might reflect upregulation of glutathione peroxide activity. Enzyme assays in an adjacent segment of spinal cord L3 showed that GP activity was greater in cases of MND than in controls ( $p<0.001$ ).

These findings may be important in relation to oxygen free radical mechanisms of neuronal injury in sporadic MND.

"CLASSIC" MOTOR NEURON DISEASE: PSYCHOLOGICAL AND SINGLE PHOTON EMISSION COMPUTED TOMOGRAPHY CORRELATES

PR Talbot, PJ Goulding, JS Snowden, D Neary, HJ Testa. Manchester Royal Infirmary, UK

"Classic" motor neuron disease (MND) is not traditionally associated with cognitive impairment, although one third of cases have evidence of frontotemporal cerebral atrophy at necropsy. The association of frontotemporal dementia (FTD) and MND has been described.

This study investigates the psychological and SPECT findings in 17 patients with classic MND. None of the patient group had clinical evidence of cognitive impairment. Psychological tests of language, perceptuospatial abilities, memory, and fronta lobe function were performed on patients and 10 controls. SPECT was performed with ${ }^{99}$ Tc-HMPAO. Mean regional cerebral blood flow indices $\left(\mathrm{rCBF}_{\mathrm{i}}\right)$ were calculated for the patient and control groups.

Psychological data showed significant group differences restricted to the scores of frontal lobe tests. SPECT group data showed significant reductions in mean $\mathrm{rCBF}_{\mathrm{i}}$ restricted to the orbitofrontal and anterior temporal cortices. This study shows subclinical evidence of frontal lobe dysfunction in classic MND. The pattern of psychological involvement and distribution of SPECT abnormality is qualitatively similar to that encountered in FTD and entirely consistent with the pathological findings in classic MND. This study reinforces the links between FTD, FTD with MND, and classic MND, which may represent a spectrum of the same disorder.

CORTICAL DYSPLASIA AND EPILEPSY

S Sisodya, A Raymond, S Free, DR Fish, SD Shorvon. Institute of Neurology and National Hospital for Neurology and Neurosurgery, London, UK

Before MRI, no cause for epilepsy could be identified in about two thirds of all cases (idiopathic or cryptogenic epilepsy). With MRI, however, structural abnormalities are found in many previously cryptogenic patients, and cortical dysplasias form an important aetiological subgroup. The term cortical dysplasias refers to a spectrum of developmental anomalies due to abnormalities of neuronal migration during foetal development or to early (usually prenatal) cerebral insults. In this case series of chronic cryptogenic epilepsy, underlying lesions are found by MRI in about $80 \%$, and cortical dysplasias account for $17 \% ; 80$ adults with epilepsy and cortical dysgenesis have now been identified, the commonest of which are subependymal heterotopias, other heterotopias, gyral anomalies (including macrogyria, polymicrogyria, lissencephaly, schizencephaly), and dysembryoplastic neuroepithelial tumours. The MRI methods that are particularly useful in detecting dysplasia include: volumetric $\mathrm{T} 1$ weighted data acquisition, the use of thin contiguous slices (<1.5 mm), multiplanar reformatting, 3D 
reconstruction, and the use of fluid attenuated inversion recovery. Other experimental techniques include fractal analysis, volume measures, and texture analysis. The cause of these conditions is largely unknown, but some (for instance subependymal heterotopia) show a striking female preponderance $(12 / 13$ cases) suggesting genetic factors; others had prenatal drug exposure. Epilepsy is often the only neurological symptom in these adult cases.

HYPEREKPLEXIA: CLINICAL AND GENETIC RELATIONSHIPS

MAJ Tijssen, R Boerman, J van Deutekom, G Padberg, RR Frants, RAC Roos, JG van Dijk. Leiden University, The Netherlands

Hyperekplexia or startle disease is an autosomal dominant disorder characterised by exaggerated startle reactions to unexpected stimuli, particularly auditory. Periodic limb movements in sleep and a pronounced head retraction reflex are additional findings. Both major and minor forms are recognised. The major form is characterised by excessive startle responses followed by stiffness, and stiffness in the neonatal period. In contrast, the minor form only shows an exaggerated startle reaction. Recently linkage was found to the markers D5S209 and D5S119 on chromosome 5q33-q35 in four pedigrees with autosomal dominant hyperekplexia. All patients in these pedigrees had the major form.

The original Dutch family has been reexamined; history was taken and neurological examination was performed. The markers D5S209, D5S119 and CSF1R were analysed in 19 patients, 26 healthy sibs, and 10 spouses.

The major form of hyperekplexia was found in 28 patients, the minor form in 16 . A locus responsible for the major form is tightly linked to the markers CSF1-R, D5S119 and D5S209 on chromosome 5q. The minor form did not show any linkage.

These linkage results show that the minor form should not be considered an integral part of hyperekplexia. The term hyperekplexia should be restricted to the major form. Although the excessive startle response is the most prominent feature of hyperekplexia, it is not the most specific one for diagnosis. The stiffness occurring in the neonatal period and in relation to startle reactions forms a very specific and more reliable sign upon which to base the diagnosis.

FINDINGS FROM THE NATIONAL SURVEY OF ELECTRONIC SCREEN GAME SEIZURES

JA Quirk, DR Fish, SJM Smith, JWAS Sander, SD Shorvon, PJ Allen. The National Hospital for Neurology and Neurosurgery, London and The National Society for Epilepsy, UK

Electronic screen game (ESG) seizures have provoked much interest but their incidence was unknown. The aim of the survey was to establish the incidence of presenting seizures provoked by ESG and separately to compare this risk with other environmental triggers in photosensitive patients. One hundred and eighteen EEG departments (90\% of the total) throughout Great Britain prospectively identified over three months all cases in whom the presenting seizure occurred while playing an ESG and all other presenting seizures and photosensitivity. A presenting seizure occurred while playing an ESG in 55 patients; 23/55 cases had unequivocal photosensitivity (all within the age range 7-19 years); 11/55 had other clinical or EEG features to suggest that the seizure may have been triggered by ESG playing. In $21 / 55$ cases no apparent causal relation could be identified, a similar number to that predicted by chance concurrence given known exposure times. When the seizure was either definitely or probably related to ESG play, most had played that and other games previously and many occurred soon after starting play. Of all new cases with unequivocal photosensitivity (143 patients), $16 \%$ were triggered by ESG, $16 \%$ by TV viewing, and $6 \%$ by other computer graphics. An annual incidence of 1.1-1.4 per 100000 for ESG triggered seizures in 7-19 year olds was estimated, representing $2-2 \cdot 5 \%$ of all epilepsies in this age group.

BEDSIDE INVESTIGATION OF NEGLECT: ONE OR MORE (SUB)SYNDROMES?

PLM de Kort, JM Minderhoud, BG Deelman, CC Tijssen. St Elisabeth and Maria Hospitals, Tilburg, and the University of Groningen, The Netherlands

The relation between neglect phenomena in patients with first ever acute hemispheric stroke was assessed.

One hundred and twenty eight patients with stroke ( 67 left; 61 right) were tested in the acute phase for the presence of several neglect phenomena. Localisation and size of the lesion and premorbid atrophy were assessed by CT.

$75 \%$ of the right hemisphere lesion group, and $61 \%$ of the left hemisphere lesion group manifested one or more neglect phenomena. All phenomena proved to be more frequent in the former group. A hierarchical order in the expression of the various phenomena was found: in nonsevere neglect only sensory inattention/ extinction and in more severe cases successively anosognosia, hypokinesia of the contralateral arm, motor extinction, disorders of the contralateral body scheme, verbal asomatognosia, and finally contralateral hypokinesia of the ipsilateral arm. In the patients who did not fully comply with the hierarchical order $(n=22)$, no alternative pattern could be ascertained. The number of neglect phenomena correlated with the size of the lesion and, independently, with premorbid atrophy. None of the phenomena was related to tension in a specific site.

It is concluded that neglect phenomena are part of one single syndrome. The severity of neglect evolves according to a hierarchical order within the phenomena of neglect. The severity of neglect is related to the size of the lesion and the degree of premorbid atrophy.

PROTON MAGNETIC RESONANCE SPECTROSCOPY IN MULTIPLE SYSTEM ATROPHY AND IDIOPATHIC PARKINSON'S DISEASE

CA Davie, GK Wenning, N Quinn, WI McDonald, CD Marsden, DH Miller. Institute of Neurology, London, UK
Multiple system atrophy (MSA) is a degenerative disease of the CNS. Clinically MSA may be confused with idiopathic Parkinson's disease (IPD) though the two conditions are quite distinct pathologically and prognostically.

We have carried out proton magnetic resonance spectroscopy (MRS) centred on the lentiform nucleus in eight patients with the predominantly striatonigral variant of MSA nine patients with IPD, and eight age matched healthy controls. Spectra were collected with a STEAM sequence at an echo time of $270 \mathrm{~ms}$.

The MSA group had significantly lower NAA/creatine ratios (mean 1.28 (SD 0.3 ) compared with controls (1.8(SD $0 \cdot 12)$ $\mathrm{p}=0.001)$. By contrast the IPD group showed normal NAA/creatine ratios $(1.79$ (SD 0.337$), p>0.5)$. The NAA/creatine ratio was significantly reduced in seven patients with MSA and only one patient with IPD. Also, the choline/creatine ratio was significantly reduced in the MSA group (1.025(SD 0.11)) compared with controls $(1 \cdot 22(\mathrm{SD} 0 \cdot 19), \mathrm{p}<0.01)$. The choline/creatine ratio showed no significant reduction in the IPD group $(1 \cdot 15, \mathrm{p}<0.5)$.

The reduction of the NAA/creatine ratio in the MSA group probably reflects neuronal loss, occurring predominantly in the putamen. The decreased choline/creatine ratio in the MSA group suggests a reduction in membrane turnover. Proton MRS is useful, non-invasive technique to differentiate MSA from IPD.

\section{Poster presentations}

CORTICAL PLAQUES AND BLOOD BRAIN BARRIER BREAKDOWN IN MULTIPLE SCLEROSIS

MD Boggild, N Haq, JR Heron, CP Hawkins. North Staffordshire Hospital Stoke-on-Trent, UK

Vascular changes are an important and early feature in the evolution of the demyelinating plaque. Whether or not blood brain barrier (BBB) breakdown is a prominent feature in cortical plaques is uncertain. Fluid attenuated inversion recovery (FLAIR) MRI sequences allow visualisation of such lesions by suppression of high signals from CSF, and gadolinium enhance ment serves to detect BBB breakdown. With these techniques, cortical, grey-white, and white matter plaques in 10 patient with clinically definite multiple sclerosis during an untreated acute relapse have been examined.

A modified FLAIR sequence (TR 3000 $\mathrm{ms}$, TI $1200 \mathrm{~ms}$, TE $150 \mathrm{~ms}$ ) was used to reduce imaging time. Nine matched slices $(5 / 7 \mathrm{~mm})$ were imaged (T2, proton density, FLAIR, and T1/contrast) per patient. For contrast scans patients received 0.1 $\mathrm{mmol} / \mathrm{kg}$ gadolinium-DTPA. Lesion site number, size, and enhancement were assessed for each slice.

BBB breakdown seems to be uncommon in cortical plaques when compared with that at the grey/white interface or within white matter. This finding is consistent with the hypothesis that myelin antigen is important in the pathogenesis of BBB breakdown An alternative, although less likely explanation, would be that myelin breakdown 
products contribute significantly to barrier breakdown.

\begin{tabular}{lccc}
\hline Lesions & $\begin{array}{l}T 2 \\
(n)\end{array}$ & $\begin{array}{l}\text { Flair } \\
(n)\end{array}$ & $\begin{array}{l}\text { Enhancing } \\
(n)\end{array}$ \\
\hline Grey & 14 & 33 & 0 \\
Grey-white & 23 & 55 & 11 \\
White & 243 & 318 & 48 \\
\hline
\end{tabular}

ABNORMAL NEURONAL MITOCHONDRIA: A CAUSE OF REDUCED N-ACETYL CONTAINING COMPOUNDS IN DEMYELINATING DISEASE RE Brenner, TE Bates, S Davies, P Munro, DN Landon, JB Clark, WI McDonald. Institute of Neurology, London UK

A frequent finding resulting from the application of proton magnetic resonance spectroscopic (MRS) techniques to the investigation of neurological diseases is a reduction in the peak assigned to $\mathrm{N}$-acetyl containing compounds (NAs). The fact that neuronal loss is a frequent pathological accompaniment has led to the suggestion that this is the cause of reduction in signal intensity. N-Acetylaspartate (NAA) is a major constituent of the peak of NA compounds, it is synthesised within mitochondria, and has been shown to be localised to neurons.

There was evidence of structural mitochondrial abnormalities in the cerebral neurons of six guinea pigs with chronic EAE, in which MRS showed a reduction in NAs. Mitochondria were then isolated from five guinea pigs with acute EAE, a model characterised by inflammation without neuronal loss or demyelination, and the NAA synthesis rate determined biochemically. It was significantly lower $(37.36(8 \cdot 79) \mathrm{nmol} /$ $\mathrm{min} / \mathrm{mg}$ protein) than values obtained from 10 control animals $(63.49(23.63) \mathrm{nmol} /$ $\mathrm{min} / \mathrm{mg}$ protein, $\mathrm{p}<0.05)$.

These findings suggest that aside from neuronal loss, a disturbance of mitochondrial function may also cause a reduction in NAs. A similar mechanism may contribute to the reduction in NAs reported in active multiple sclerosis plaques particularly when it is reversible.

NUCLEAR MAGNETIC RESONANCE PROTON SPECTROSCOPY OF MENINGIOMAS RE Brenner, CA Davie, ND Kitchen, DGT Thomas, DH Miller, WI McDonald. Institute of Neurology, London UK

The preoperative identification of cerebral tumours remains a challenge. Proton magnetic resonance spectroscopy (MRS) detects at least nine cerebral metabolites, and is being increasingly used to investigate the biochemical changes accompanying neurological disease. The information obtained is often non-specific, however, providing perhaps prognostic data, but rarely a diagnosis.

In vivo short echo proton spectra (TE $=$ $10 \mathrm{~ms}$ ) obtained at $1.5 \mathrm{~T}$ from two patients with cerebral meningiomas showed an increase in the peak assigned to choline containing compounds, a reduction in that assigned to $\mathrm{N}$-acetyl containing compounds, and a prominent resonance assigned to alanine, as has previously been described. Also, a pronounced increase was seen in the peaks assigned to glutamate and glutamine. This was confirmed by high field spectra $(11.5 \mathrm{~T})$ obtained from tumour extracts. High concentrations of glutamate and glutamine have not previously been reported in cerebral tumours. Although this finding clearly requires confirmation, it raises the possibility that MRS may provide a "metabolic fingerprint" and thus aid in the non-invasive diagnosis of cerebral space occupying lesions.

RESPIRATORY ABNORMALITIES DUE TO CRANIOVERTEBRAL JUNCTION COMPRESSION HC Chandler, RS Howard, HA Crockard, NP Hirsch, F Henderson, JM Stevens. The National Hospital for Neurology and Neurosurgery, London UK

Apnoea and sudden death are known to occur as a consequence of medullary compression. Lesser respiratory abnormalities may be associated with foramen magnum lesions, which cause brainstem compression. The presence of nocturnal hypoventilation may indicate the potential for perioperative respiratory difficulties. Thirty eight patients with severe craniovertebral compression (12 rheumatoid, 14 congenital platybasia/Klippel-Feil, six osteogenesis imperfecta, five tumours, one odontoid fracture) were studied with overnight pulse oximetry and subsequent computer analysis. Overnight baseline oxygen saturation $\left(\mathrm{SpO}_{2}\right)$ and the number of dips in $\mathrm{SpO}$ lasting longer than 10 seconds were calculated per hour of recording. All patients underwent multiplanar CT/myelography or MRI with an assessment of craniovertebral angles and qualitative assessment of medullary and spinal cord compression. Rheumatoid patients had the greatest respiratory abnormalities, but only when the medulla was deformed by a translocated odontoid peg. Nocturnal respiratory abnormalities were rare in patients with other lesions at the craniocervical junction. No correlation was found between the severity of the lower cranial nerve palsies and the presence of respiratory abnormalities. These studies suggest that clinically unsuspected respiratory abnormalities may occur in patients with severe medullary compression, especially when due to rheumatoid arthritis. Nocturnal oximetry may be used to detect these abnormalities, possibly identifying patients prone to postoperative respiratory complications.

MOTOR NERVE CONDUCTION VELOCITIES CALCULATED BY F TACHEODISPERSION IN PATIENTS WITH ANTERIOR HORN CELL DISEASE

E Chroni, RS Howard, CP Panayiotopoulos, GT Spencer. Guy's and St. Thomas' Hospital, London, UK

F Tacheodispersion, a neurophysiological technique based on $F$ waves, was used to determine the motor nerve conduction velocities in 16 patients with motor neuron disease and 19 patients with sequelae after paralytic poliomyelitis. The findings were compared with two age matched groups, each consisting of 20 healthy volunteers. In each subject the $F$ wave maximal, minimal, and mean conduction velocities and the difference between maximal and minimal velocities of the ulnar and peroneal nerves were calculated. M Response distal latency, amplitude, and conduction velocity were also measured. There was a statistically significant slowing of one or more of the $F$ wave estimated velocities in the absence of $M$ response abnormalities in $31.3 \%$ of nerves in the motor neuron disease group and in $27.3 \%$ of nerves in the poliomyelitis group; between $6.3 \%$ and $37.5 \%$ of the nerves studied showed a significant change in the difference between maximal and minimal conduction velocities. These results favour the presence of axonopathy in some patients with anterior horn cell disease.

CAROTID TIAS: SHOULD THE ANATOMICAL TERRITORY BE WIDENED?

PN Cooper, J Hobbs, PRD Humphrey. The Walton Centre for Neurology and Neurosurgery, Liverpool, UK

Two patients with carotid dissection are reported, in whom clinical assessment was misleading, and suggested that the symptoms had arisen in the vertebrobasilar territory.

A 37 year old man developed tingling on the right of his neck, with loss of central vision in both eyes for 30 minutes. The next day both legs felt heavy, and he felt as if drunk. CT showed an infarct in the right occipitoparietal region. Carotid angiography showed a right internal carotid dissection. There was also an occlusion in the right posterior cerebral artery.

A 40 year old man suddenly developed headache with altered sensation in his right leg, lasting several hours. The next day he woke with loss of his right visual field. On examination he had a macular sparing right homonymous hemianopia. CT showed an infarct in the medial aspect of the left occipital lobe; Doppler scan and an MRI angiogram showed a stenosis in the left internal carotid. Subintimal thrombus was found on conventional MRI.

The vertebral arteries were normal in both patients.

Stroke in posterior cerebral artery territory can result from internal carotid artery disease; it is important to be aware of this when assessing patients with cerebrovascular disease.

ELECTROPHYSIOLOGICAL STUDIES IN BOTULISM

EMR Critchley, JD Mitchell, Y Fahim, HN Gibson. Royal Preston Hospital, UK

Twelve of 27 patients with type B botulism were investigated electrophysiologically during the June 1989 outbreak from infected hazelnut yoghurt.

The three most severely affected patients presented before the moderately affected patients - as inevitably happens in most outbreaks. They rapidly developed a flaccid tetraplegia and initially had extensor plantar responses. EMGs failed to show pathognomonic incremental responses and low compound muscle action potentials (CMAPs) were suggestive of conduction block of peripheral neuropathic type. This led to some diagnostic confusion in the early stages.

As the outbreak progressed more classical findings were obtained and the neuromuscular transmission defect was best shown by 
postcontraction facilitation of the amplitude of compound muscle action potentials. Upper limb somatosensory evoked potentials in seven patients were entirely normal even though three patients showed lower limb pyramidal signs.

LONG TERM MORBIDITY AFTER BOTULISM EMR Critchley, JD Mitchell, AL Burton. Royal Preston Hospital, UK

Thirty months after an outbreak of foodborne botulism, after the completion of legal proceedings, all 26 surviving patients were examined by questionnaire. Twenty one had been personally interviewed in the previous year. Sixteen of the 26 patients reported prolonged symptoms.

Five adults with prolonged symptoms, representative of the middle age range, had a further examination with blood tests, EMGs, respiratory function tests, and a psychological questionnaire developed for investigation of the chronic fatigue syndrome. These tests were essentially normal.

EFFECTS OF INTRAVENOUS

METHYLPREDNISOLONE ON LYMPHOCYTE DISTRIBUTION AND FUNCTION IN PATIENTS WITH MULTIPLE SCLEROSIS

AD Crockard, M Treacy, AG Droogan, TA McNeill, SA Hawkins. Royal Victoria Hospital, Belfast, UK

Intravenous methylprednisolone (IVMP) is the most effective known treatment for relapses of multiple sclerosis but the mechanism is unclear. Ten patients in relapse of clinically definite multiple sclerosis were treated with a five day course of IVMP (500 mg daily). Circulating lymphocyte subpopulations and determination of mitogen induced $\tau$-interferon ( $\tau$-IFN) production, were performed immediately before initiation of treatment (day 1), during treatment (24 hours after first dose, day 2), and at 24 hours and one week after treatment (days 6 and 12). Significant reductions in CD3+, CD4+, CD8+, CD4CD45RA+, and $\mathrm{CD} 4 \mathrm{CD} 45 \mathrm{RO}+$ subpopulations were noted within 24 hours of the start of treatment. These changes had returned to normal by day 6 and remained normal on day 12. Despite the reduction in total $T$ cell numbers during treatment, the $\tau \delta \mathrm{T}$ cell subpopulation was not significantly altered. Expression of HLA-DR on B cells and monocytes declined transiently on day 2 to about $50 \%$ of pretreatment values. Production of $\tau$-IFN was significantly reduced $(\mathrm{p}<0.05)$ during treatment, but had returned to pretreatment levels by day 6 (day 1:2148 (SD 656) IU/ml; day 2:1068 (SD 470) IU/ml; day 6:2424 (SD 1158); day 12:1940 (SD 978) $\mathrm{IU} / \mathrm{ml}$ ). Decreased $\tau$-IFN production may play a part in the rapid onset of improvement that occurs after IVMP treatment.

PROTON MAGNETIC RESONANCE SPECTROSCOPY IN ADULT CASES OF PHENYLKETONURIA

CA Davie, GJ Barker, D Brenton, DH Miller, AJ Thompson. Institute of Neurology, London, UK
Neurological deterioration and abnormalities on MRI may occur in adults with phenylketonuria (PKU) in whom diagnosis is delayed or who have poor dietary control. Proton magnetic resonance spectroscopy (MRS) has been carried out on five adult patients with PKU and abnormalities on MRI and in eight healthy, age matched controls. MRI showed diffuse high signa abnormalities on T2 weighted images in all patients. Spectra collected at echo times of 10 and $135 \mathrm{~ms}$ showed normal NAA creatine ratios $(2.2$ (SD 0.31$)$ ) in areas of MRI abnormality and from areas of norma appearing white matter (NAWM) compared with controls (1.88 (SD 0.18) Choline/creatine ratios were increased in the MRI lesions (1.16 (SD 0.3)) and in areas of NAWM (1.14 (SD 0.1) compared with control white matter $(0.92$ (SD 0.07)) Inositol/creatine ratios were reduced in lesions $(0.47$ (SD 0.2)) relative to contro white matter $(0.62$ (SD 0.2)). No abnormal peaks attributable to phenylalanine or myelin breakdown products were seen.

The preservation of the NAA/creatine ratio suggests that little axonal loss is occurring within the MRI visible abnormalities in PKU whereas the raised choline/creatine ratio suggests increased membrane synthesis. The absence of myelin breakdown products also suggests that active myelin breakdown is not prominent.

The MRS findings in PKU support a non-destructive pathological process with increased membrane/myelin turnover.

LIAISON NEUROLOGY: INFLUENCE OF OBTAINING A NEUROLOGICAL OPINION ON THE DIAGNOSIS AND MANAGEMENT OF HOSPITAL INPATIENTS

TP Enevoldson, L Ginsberg, SR Hammans, MJ Steiger. Royal Free Hospital, London, UK

Referrals of inpatients in a multidisciplinary teaching hospital to the neurological service were monitored for six months. Of 169 different patients seen in total during this period, $91(54 \%)$ had the diagnosis of the referring practitioners confirmed by neurologists. The initial diagnosis was, however, changed by a neurologist in $18 \%$ of cases and a completely new diagnosis was made in another $15 \%$, where there had been no pre-existing diagnosis. In $14 \%$ of the patients, the diagnosis remained unclear even after neurological assessment. The commonest groups of disorders seen in the diagnosed patients were cerebrovascular ( $17 \%$ of total), neuromuscular $(15 \%)$, and metabolic/toxic encephalopathies (10\%). The time honoured image of neurologist as pure diagnostician is belied by the fact that neurological consultation led directly to a change in drug treatment or to surgical intervention in 52 cases (31\%).

A STUDY OF PATIENTS WITH MULTIPLE SCLEROSIS BY MAGNETIC RESONANCE SPECTROSCOPY

JAG Geelen, AR Peters, JM Minderhoud, EJ's-Gravenmade. University of Groningen, The Netherlands

Magnetic resonance spectroscopy (MRS) was used to detect abnormalities of white matter and lesions in patients with multiple sclerosis and to examine the relation between disability (EDSS) and the biochemical parameters obtained with MRS.

MRS and two dimensional spectroscopic imaging were performed on a Philips 1.5 T S15/ACS MR imager. The nominal voxel size was $0.84 \mathrm{ml}$. The levels of $\mathrm{N}$-acetylaspartate (NAA), cholines, and creatines were each expressed as fractions of the sum of these substances. The data of all voxels containing normal white matter (NWM, controls), normal appearing white matter (NAWM, patients), or multiple sclerosis lesions were pooled and these three datasets were compared. Furthermore, the average signal intensities of NAA, cholines, and creatines were measured in individual patients.

Eleven patients (four relapsing-remitting, five secondary progressive, two primary progressive) and three healthy controls were studied. The NAA fractions in pooled voxels of NWM were significantly higher $(p<0.001)$. Reduction of NAA is considered to be indicative of axonal and neuronal loss. The following findings were interesting: there was a significant correlation between the disability (EDSS) of individual patients and their ratio of NAA in NAWM and in lesions. A similar result appeared when the relapsing-remitting and secondary progressive patients were compared. The latter phenomenon does not indicate a fundamental biochemical difference between the two types of multiple sclerosis but reflects the increased EDDS in secondary progressive patients.

REMOTE MEMORY IN EARLY ALZHEIMER'S DISEASE: A CROSS SECTIONAL STUDY JDW Greene, A Baddeley, JR Hodges. University of Cambridge and MRC Applied Psychology Unit, UK

Amnesia is the earliest cognitive disturbance in dementia of Alzheimer type (DAT). Although the nature of the anterograde amnesia is well documented, the pattern of remote memory impairment has been less clearly defined especially in early disease and studies to date have produced conflicting results. Remote memory was investigated with a range of tests in 28 patients with minimal to mild DAT (MMSE scores 13-29) and 17 age and IQ matched controls.

The Autobiographical Memory Interview showed impaired memory for personal life episodes, which differentially affected the more recent past (a temporal gradient). On a famous faces test, recognition of faces was preserved but naming was severely impaired even in the minimally demented subjects. Again there was evidence of a temporal gradient. A famous names test showed a similar pattern - that is, preserved recognition of names as famous from among three foils but impaired generation of semantic information about the same names.

These findings show that remote memory is affected very early in the course of DAT and that certain subcomponents within this domain show selective impairment. The temporal gradient seen in early disease may reflect selective perihippocampal pathology; the subsequent flattening of the gradient as semantic memory becomes more severely affected possibly relates to temporal neocortical involvement. 
ISOLATION AND CHARACTERISATION OF A HUMAN CELL LINE OF THE

OLIGODENDROCYTE TYPE 2 ASTROCYTE LINEAGE DERIVED FROM A GLIOBLASTOMA MULTIFORME

NJ Gutowski, K Bevan, $M$ Noble (introduced by JR Heron). University College London, UK

There is little evidence of the extent to which the studies of rodent glia are relevan to human cells. Despite the large body of research on CNS rodent glial cell precursors and their biological properties, no clear correlation has been established between glial lineages and particular human glial tumours. By growing human glioma samples with techniques developed to study rodent glial precursor cells, a glioblastoma multiforme derived population ( $\mathrm{Hu}-0$ $2 \mathrm{~A} / \mathrm{Gbl}$ ) has been grown in vitro that corresponds closely with oligodendrocyte type 2 astrocyte (0-2A) progenitor cells of the rodent $\mathrm{CNS}$. $\mathrm{Hu}-0-2 \mathrm{~A} / \mathrm{Gbl}$ cells resemble $0-2 \mathrm{~A}$ progenitors in antigenic phenotype and in response to various biological stimuli and have continued to generate oligodendrocytes and astrocytes for more than 15 passages in vitro. The metabolite composition of $\mathrm{Hu}-0-2 \mathrm{~A} / \mathrm{Gbl}$ cells determined by 'H-NMR analysis, showed a spectrum that previous studies suggest may be uniquely expressed by $0-2 \mathrm{~A}$ progenitors. These results indicate that the human $\mathrm{CNS}$ contains $0-2 \mathrm{~A}$ progenitor-like cells that can contribute to the most malignant family of human brain tumours and further suggests that at least two distinct glial lineages can give rise to glioblastoma multiforme.

SURVEY OF MONITORING FOR HEPATIC OR HAEMATOLOGICAL ABNORMALITIES DURING CARBAMAZEPINE TREATMENT

B Henderson, JM Bamford, H Remmington. St James's University Hospital, Leeds, UK

Data sheet recommendations suggest checking monthly full blood counts (FBCs) and periodic liver function tests (LFTs) during chronic carbamazepine treatmen (which may double the monthly cost of therapy) although carbamazepine is not associated with severe agranulocytosis.

A tick questionnaire postal survey of 281 members of the Association of British Neurologists was conducted in an attempt to assess current practice in monitoring for hepatic and haematological abnormalities during carbamazepine treatment.

Three questions with four responses ("never", "rarely", "often", and "routine" or "never", "once", "twice", or "more") relating to checking a full blood count (FBC) or liver function tests (LFTs) and one question on serious problems encountered during carbamazepine treatment were asked. There were 187 completed questionnaires from 189 returns $(67 \%)$. Results were as follows: FBC/LFTs: before treatment $42 \% / 45 \%$ never, $31 \% / 31 \%$ rarely $15 \% / 13 \%$ often, and $11 \% / 11 \%$ as a routine checked an FBC or LFTs. In the first month of treatment $78 \% / 82 \%$ never $21 \% / 17 \%$ once, and $0.5 \% / 0.6 \%$ twice checked FBC/LFTs. After the first month $75 \% / 78 \%$ never, $13 \% / 12 \%$ once, and $3 \% / 3 \%$ twice checked an FBC or LFTs.

Thirty six per cent had encountered a serious problem relating to an abnormal FBC and $20 \%$ to LFTs.
It is concluded that most $(>75 \%)$ neurol ogists in this survey do not routinely monitor for hepatic or haematological abnormalities during carbamazepine treatment suggesting that the indications for monitoring long term treatment should be re-examined.

CONTROL OF RESPIRATION IN THE “LOCKED IN SYNDROME"

P Heywood, RS Howard, K Murphy, D Corfield, M Morrell, A Guz. Charing Cross Hospital and National Hospital for Neurology and Neurosurgery, London, UK

Respiratory regulation by the CNS involves automatic control from medullary respiratory centres and voluntary control via corticospinal pathways. The "locked in syndrome" (LIS) isolates medullary respiratory centres from rostral influences passing in the ventral pons with the corticospinal tracts. Respiratory variables during resting wakefulness were studied in a 44 year old man with LIS due to bilateral ventral pontine infarction five months postictus when he was breathing spontaneously via a tracheostomy. Results were compared with data from five tracheotomised control subjects. Variability about the mean in the patient with LIS was significantly less than in controls for $T_{I}, T_{E}, V_{t}$, and $P_{E T} C O_{2}$ Ventilatory sensitivity to $\mathrm{CO}_{2}$ was $1.71 / \mathrm{min}$ $\mathrm{mm} \mathrm{Hg}$ (normal $1 \cdot 5-3 \cdot 8$ ). In the patient with LIS the mean resting $\mathrm{P}_{\mathrm{ET}} \mathrm{CO}_{2}$ was 39.0 $\mathrm{mm} \mathrm{Hg}$ and prolonged apnoea occurred when $\mathrm{P}_{\mathrm{ET}} \mathrm{CO}_{2}$ was $<38.0 \mathrm{~mm} \mathrm{Hg}$. Although completely unable to regulate respiratory muscle activity voluntarily, when he was amused he laughed and altered his respiratory rate and volume.

These findings confirm that "isolated" human respiratory centres produce a very regular respiratory rhythm upon which variability is superimposed by separate descending systems mediating volitional and emotional control. They also show that medullary respiratory centres retain normal sensitivity to $\mathrm{CO}_{2}$ and that resting $\mathrm{P}_{\mathrm{ET}} \mathrm{CO}_{2}$ is held extremely close to the apnoeic threshold.

MITOCHONDRIAL ENCEPHALOMYOPATHY, LACTIC ACIDOSIS, AND STROKE-LIKE EPISODES (MELAS) IN ADULTS: A REPORT OF THREE CASES AND A REVIEW OF THE

LITERATURE

JAP Hiel, A Verrips, FJM Gabreëls, A Keyser, PLJA Bernsen, W Ruitenbeek, $\mathrm{R}$ Wong Chung, D de Vries, BA van Oost, $P$ Wesseling. University Hospital of Nijmegen, The Netherlands

In general the first manifestations of the MELAS syndrome occur in early childhood (early onset MELAS), but onset at adult age (late onset MELAS) has been described in several studies. In this study the clinical and laboratory findings in late onset MELAS (LOM) is compared with those in early onset MELAS (EOM).

Late onset is defined by an age of 16 years or older. Three patients with LOM are presented. The clinical and laboratory findings of 27 patients with LOM are compared with those of 55 patients with EOM by review of the literature. Clinical and laboratory features in LOM closely resembled those in EOM, an exception being the genetic findings. In 13 patients with LOM and in 16 patients with EOM a point mutation at nucleotide position 3243 in muscle mitochondrial DNA was present. The amount of mutated mtDNA was determined in 10 out of the 13 and in 11 out of the 16; it ranged from 50 to $95 \%$ in LOM and 56 to $95 \%$ in EOM, the overall amount of mutated mtDNA being significantly lower in $\operatorname{LOM}(p=0.03 \mathrm{t}$ test).

It is concluded that LOM differs from EOM only in a genetic aspect with lower amounts of mutated mtDNA in LOM than EOM. These findings suggest that the age of onset may depend on the amount of mutated DNA.

LUMINANCE AND CHROMATIC CONTRAST SENSITIVITY IN ANTERIOR ISCHAEMIC OPTIC NEUROPATHY

W Honan, G Naylor. The Midland Centre for Neurosurgery and Neurology, Birmingham, UK

A computer based system was used to measure luminance and chromatic spatial contrast sensitivity. Red and green sine wave gratings, of about equal luminance, produced a luminance (in phase) stimulus and a chromatic (antiphase) stimulus. The stimulus field was circular, $8^{\circ}$ diameter, and mean luminance $35 \mathrm{~cd} \mathrm{~m}^{-2}$. It was presented on a uniform yellow, rectangular background field of equal luminance, $10^{\circ}$ vertical by $14^{\circ}$ horizontal.

Ten cases of anterior ischaemic optic neuropathy (AION), were studied (median Snellen acuity 6/12, range 6/5-6/36) and compared with an age matched control group (median acuity 6/6, range 6/5-6/9). Fixation was central. Uniocular contrast sensitivity measurements were made, by the method of limits, at 1,4 , and $16 \mathrm{c} / \mathrm{deg}$.

The results for chromatic contrast sensitivity showed mean losses in the AION group compared with the control group of $0.95 \mathrm{log}$ units at $1 \mathrm{c} / \mathrm{deg}(\mathrm{p}<0.001), 1.02$ $\log$ units at $4 \mathrm{c} / \mathrm{deg}(\mathrm{p}<0.001)$, and 0.49 $\log$ units at $16 \mathrm{c} / \mathrm{deg}(\mathrm{p}<0.001)$. For luminance contrast sensitivity mean losses in the AION group were $0.94 \log$ units at $1 \mathrm{c} / \mathrm{deg}$ $(\mathrm{p}<0.001), 1.09 \log$ units at $4 \mathrm{c} / \mathrm{deg}(\mathrm{p}<$ $0.001)$, and $0.42 \mathrm{log}$ units at $16 \mathrm{c} / \mathrm{deg}$ $(\mathrm{p}<0.01)$. The results indicate a uniform loss of chromatic and luminance contrast sensitivity in AION.

CLINICOPATHOLOGICAL FINDINGS IN 22 CASES OF "LEWY BODY DISEASE"

ENH Jansen, RAI de Vos, FC Stam, R Ravid, DF Swaab, H Braak, D Enschede, and the Institute for Brain Research, Amsterdam, The Netherlands and the University of Frankfurt, Germany

Postmortem neuropathological findings in 22 consecutive cases of Parkinson's disease are reported. The incidence of cortical Lewy bodies (CLBs) and/or Alzheimer type pathology (ATP) in specific areas of the brainstem, neocortex and allocortex is correlated with clinical findings of depression, changes in cognitive state, and neurobehavioural abnormalities. Lewy bodies of variable density and localisation were found in the neocortex of all subjects. ATP was found in 14 subjects but a diagnosis of 
Alzheimer's disease on the basis of the Khachaturian and CERAD criteria could be made in only five. It was possible to stage the Alzheimer's disease changes according to the Braak and Braak criteria in no more than 17 subjects. The three subjects in the isocortical stage all had clinical dementia, but this was present in only four of the six subjects in limbic stages III and IV. Two subjects in whom clinical dementia was associated with CLBs had no trace of ATP.

Although the number of patients in the study was small, the existence of a specific clinicopathological entity in patients with Parkinson's disease and CLBs could not be confirmed.

NO ASSOCIATION OF MULTIPLE SCLEROSIS WITH HLA DR AND DQ ALLELES IN SHANGHAI CHINESE

MA Kelly, Y Zhang, MA Penny, KH Jacobs, DA Cavan, CH Mijovic, KY Chou, AH Barnett, DA Francis. University of Birmingham, UK

The association of multiple sclerosis with the human leucocyte antigen (HLA) class II loci DR and DQ was investigated in 48 patients from Shanghai, China and 105 patients of British Caucasian origin, to determine whether common alleles predispose to the disease in both races. In the Caucasians, multiple sclerosis was significantly positively associated with the DRB1 * 1501. DQA1 * 0102. DQB1 * 0602 haplotype. No DR or DQ allele differed significantly in frequency between the patients and the controls of the Chinese population. These data suggest that $(a)$ the disease may have a different immunogenetic background in the two populations; (b) the environmental factors involved in triggering the disease in each race may interact differently with the HLA molecules encoded by the DRB1 ${ }^{\star} 1501$. DQA1 ${ }^{\star} 0102$. $\mathrm{DQB1}{ }^{\star} 0602$ haplotype; (c) the Chinese DRB1 ${ }^{\star} 1501$ haplotype may carry an allele that protects against multiple sclerosis, masking the predisposing influence of the DR and DQ loci; or (d) the DR and DQ associations in the Caucasian population are secondary to linkage disequilibrium with a true susceptibility allele, which is not found on the Chinese DRB1`1501 haplotype.

A PROSPECTIVE STUDY OF THE EFFECTIVENESS OF NEUROREHABILITATION IN MULTIPLE SCLEROSIS

D Kidd, AJ Thompson. Institute of Neurology, London, UK

Whereas inpatient rehabilitation may improve both disability and handicap in patients with multiple sclerosis, the duration of benefit is uncertain. To assess this, a prospective study incorporating outcome measures was carried out.

Forty seven patients (five relapsing/remitting, 42 progressive) with definite multiple sclerosis undergoing a two to three week rehabilitation programme were studied. Patients were assessed by a single observer on admission, discharge, and after an interval of three months with Kurtzke's Expanded Disability Status Scale (EDSS), the Functional Independence Measure (FIM), and the Environmental Status Scale (ESS).
The mean FIM and ESS improved from 62 and 19 on admission to 72 and 17 respectively at discharge. $90 \%$ and $47 \%$ of patients improved on the FIM and ESS respectively during their stay. Forty four patients were re-examined after three months; $62 \%$ had maintained or increased their functional abilities. The mean FIM and ESS was 70 and 17 respectively. 19 patients showed some neurological deterioration, of whom 10 suffered relapse, although only two showed deterioration on the EDSS. The mean change in FIM and ESS in those who had deteriorated was -6.2 and -0.4 respectively, and 0.2 and 1.0 in those who were unchanged.

In summary, whereas a third of patients had further clinical activity resulting in a deterioration in disability and handicap, function was maintained in most patients three months after discharge.

MAGNETIC RESONANCE IMAGING DYNAMICS OF PROGRESSIVE MULTIPLE SCLEROSIS

D Kidd, AJ Thompson, BE Kendall, DH Miller, WI McDonald. Institute of Neurology, London, UK

The pathogenesis of progressive multiple sclerosis is unknown. To investigate how such patients change over time, a serial MRI study of the brain and spinal cord was performed.

Nineteen patients (10 primary progressive, nine secondary progressive) underwent monthly brain and spinal cord MRI, with and without gadolinium enhancement, and clinical examination for 12 months. Active MRI lesions were defined as new, enlarging, or newly enhancing lesions.

The mean EDSS initially was 4.9 and 5.4 in primary and secondary progressive groups respectively and $6 \cdot 1$ and $6 \cdot 2$ after 12 months. In the secondary progressive group there were five relapses in four patients. In total, 110 active brain lesions developed in the secondary progressive group and 19 in the primary progressive group. In the spinal cord three active lesions were noted in each group.

Thus in using MRI to detect active lesions during treatment trials, spinal examination contributes little.

PERONEAL NEUROPATHY IN PATIENTS WITH CANCER: A PARANEOPLASTIC SYNDROME? PJ Koehler, M Buscher, C Rozeman, A Twijnstra. Hospital De Wever and Gregorius, Heerlen and University Hospital Maastricht, The Netherlands

A retrospective study was performed to investigate the occurrence of peroneal neuropathy $(\mathrm{PN})$ in patients with cancer, as well as that of cancer in patients with PN.

Clinical, neurographical, and myographical data of patients with PN, in two neurological clinics during a five year period (1988-92) were analysed. Patients with cancer were selected. Automated databanks from the pathology department were consulted to find additional patients with PN and with cancer. A tumour registration databank was consulted for epidemiological data of the area.

The population served by the two clinics numbers 410000 people and 8370 patient were diagnosed to have a new cancer. Of a total of 355 patients with PN, 73 had cancer, in 53 supposed to be related to cancer. Thus 53 of $8370(6.5 \%)$ patients with cancer had PN, whereas in non-cancer patients (401630) PN occurred in $302(0 \cdot 7 \%)$. Otherwise, 53 of 355 (14.9\%) patients with PN had cancer whereas 8317 of $409645(2 \%)$ of patients without PN had cancer. Cancer was mostly present in elderly men with PN. Weight loss was established in $35 / 53$ patients, but in 14/53 it was unknown. Chemotherapy did not play a causal part. In some patients PN preceded the diagnosis of cancer.

It is concluded that $\mathrm{PN}$ occurs relatively often in patients with cancer and has not been found to be part of a polyneuropathy; it was related to weight loss. Cancer more often occurs in patients with than without PN, particularly in elderly men, even if corrected for the relation between age and cancer. PN in patients with cancer is supposed to be due to a combination of metabolic and mechanical factors. The results justify a prospective study on the relation between $\mathrm{PN}$ and cancer.

THREE CASES WITH STATUS EPILEPTICUS DURING VIGABATRIN TREATMENT

MCTFM de Krom, N Verduin, E Visser, M Kleijer, F Scholtes, JHM de Groen. Maastricht University Hospital, The Netherlands

Vigabatrin ( $\gamma$-vinyl-GABA or GVG) is used in refractory epileptic seizures, particularly complex partial fits. Due to irreversible inhibition of $y$-aminobutyric acid transaminase (GABA-T) GVG increases the brain concentration of the inhibiting neurotransmitter GABA. Three patients are reported who developed a severe status epilepticus (SE) during GVG treatment.

Case I: A 17 year old woman with a spastic tetraparesis, generalised epilepsy and myoclonic jerks, and moderate mental handicap had two previous episodes of SE. Another episode of SE followed introduction of GVG (200 mg/day), cessation of DPH and tapering of PB. GVG was tapered in five days whilst thiopentone was administered over a period of 21 days. On PB and CZP she slowly regained consciousness but some myoclonic jerks remained; she was worse than before admission. Nerve conduction tests showed axonal polyneuropathy. She died due to probable pneumonia. A tentative diagnosis of neuroaxonal dystrophy could be made, necropsy was not performed.

Case II: A 16 year old woman had partial seizures after surgery for a frontoparietal fibrosarcoma and radiotherapy. After several years treatment with DPH and CBZ she relapsed. DPH was replaced by GVG and CBZ increased. A few months later she developed SE, which recurred daily for 10 days. GVG was stopped after five days. Four days later she had only minor frequent seizures on DPH, CBZ, and CZP.

Case III: A 33 year old, mentally handicapped, female patient had tonic-clonic and complex partial seizures from age 4. On oxcarbazepine (OCB) and clobazam she had seizures on average $0.5-1.0$ per 24 hours. GVG was introduced and OCB tapered. While on $2000 \mathrm{mg} \mathrm{GVG}$ and 300 mg OCB, she developed a generalised convulsive SE lasting 13 hours and stopped by 
iv infusion of propofol; GVG was discontinued.

Exacerbation of seizures has been described occasionally for DPH, CBZ, and benzodiazepines. SE during GVG treatment could either be a coincidence or due to a possible disinhibiting effect of GABA in the nigrocollicular pathway by GVG.

SERIAL MONTHLY GADOLINIUM ENHANCED MAGNETIC RESONANCE IMAGING IN THE MONITORING OF TREATMENT OF MULTIPLE SCLEROSIS: THE ANTI-CD4 TRIAL DESIGN M Lai, $\mathrm{R}$ van Oosten, $\mathrm{F}$ Barkhof, $\mathrm{CH}$ Polman, S Hodgkinson, N Lewellyn-Smith, L Cuzner, AJ Thompson, DH Miller, WI McDonald. Institute . of Neurology, London, UK; Free University Hospital, Amsterdam, The Netherlands; and University of Sydney, Sydney, Australia

Based on data from serial monthly gadolinium enhanced brain MRI of 23 untreated patients with multiple sclerosis, sample sizes have been calculated for a treatment trial in which reduction in MRI activity is the primary measure of outcome. In a study of six months duration with a parallel group, placebo controlled design, a $70 \%$ reduction in the number of active lesions was seen with a greater than $90 \%$ power in $2 \times 40$ patients.

Because such a study requires far fewer patients and shorter follow up time than phase three clinical studies it is an excellen way of screening new treatments. It is not definitive, however, because of the uncertain relation between changes in MRI and long term clinical outcome.

This protocol is currently being used in the study of monoclonal CD4 antibodies. Seventy two patients with clinically active multiple sclerosis after either relapsing emitting or secondary progressive course have been randomly allocated to receive either $50 \mathrm{mg}$ of chimaeric CD4 antibody (Centocor, cM-T412) or placebo monthly for six months. Active treatment resulted in a pronounced and rapid reduction in the peripheral blood CD4-count, which subsequently increased, although it remained below the normal range for at least a number of months after treatment. MRI evaluation will be in the second half of 1994 .

DOMINANT MIGRATORY NEUROPATHY WITH CHROMOSOME 17 DELETION

BRF Lecky, PK Thomas, J Tyson, S Malcolm. Walton Centre, Liverpool and Royal Free Hospital School of Medicine, and Institute of Child Health, London, UK

A 66 year old man had had brief scalp pains since his 20 s, often with an electric shocklike quality. In his late 50 s he developed migratory trunk and limb pains, at times severe, in variable and localised areas but sparing the extremities. Episodes lasted between several hours and a few days with prolonged symptom free intervals. The pain was not associated with numbness and episodes were not provoked by stretch or trauma. The patient's father and sister are similarly affected and a son and daughter have developed scalp pains in their $30 \mathrm{~s}$

Neurological signs were confined to loss of tendon reflexes and mild impairment of 2 point discrimination. Motor conduction velocities were normal. Sensory action potentials were very small with normal or mildly reduced sensory conduction velocities. Sural nerve biopsy showed reduction in the number of small myelinated axons. Examination of CSF was normal.

Analysis of DNA showed a chromosome $17 \mathrm{p}$ deletion, which is now known to be the basis for hereditary liability to pressure palsies. It is becoming clear that phenotypic expression of this condition is varied and unexpected. Although this family shows superficial similarity to Wartenburg's syndrome, this condition is sporadic and is not known to have autosomal dominant inheritance.

AXON FLARE RESPONSE IN PERIPHERAL NEUROPATHIES

AC Mann, GA Jamal. Southern General Hospital, Glasgow, UK. (Introduced by JP Ballantyne)

The axon flare response is a neurally mediated rise in skin blood flow that occurs secondary to a noxious stimulus. Current evidence suggests that it is largely dependent on the integrity of the polymodal nociceptive group $\mathrm{C}$ nerve endings and therefore gives a potential method by which this group of fibres can be assessed. This is mportant as current routine neurophysiological tests fall short of such an assessment. The purpose of this study was to compare the axon flare response as measured by lase Doppler flowmetry in normal controls and patients with peripheral neuropathies. The flare was induced by applying standardised electrical pulses to the dorsum of the hand and foot. Twelve age and sex matched subects were included in each group and patients with diabetes mellitus were excluded due to their risk of vascular disease. The area of the lower limb response was significantly reduced in the neuropathy group $244 \mathrm{~V} / \mathrm{min}$ ) compared with the controls $(1099 \mathrm{~V} / \mathrm{min}) ; \mathrm{p}<0.05)$. No significant dif ference was found in the upper limb responses. These results suggest that this technique will be of use in the study of various neuropathic conditions and further work is indicated particularly in patients in whom pain is a prominent symptom.

TRANSCRANIAL COLOUR IMAGING AND "ULTRASOUND ANGIOGRAPHY" OF CEREBRAL ARTERIOVENOUS MALFORMATIONS

PJ Martin, RJ Abbott, P Critchley, IF Pye, PAH Millac. Leicester Royal Infirmary, UK

Transcranial colour coded sonography (TCCS) is a recent development in noninvasive cerebral vascular imaging. It allows visualisation of the brain parenchyma using B-mode ultrasound and imaging of the basal cerebral arteries with colour flow ultrasound. Pulsed wave Doppler enables measurement of blood flow velocities. TCCS was used to evaluate the cerebral circulation in 12 patients with known arteriovenous malformation (AVM)

Nine AVMs (75\%) were successfully imaged (Spectra, Diasonics Inc), three being visualised with "ultrasound angiography". Principal AVM feeder vessels were identified in 11 cases (seven middle cerebral artery, two posterior cerebral artery, one anterior cerebral artery, one basilar artery).
Flow in these vessels was characterised by raised velocities compared with the equivalent contralateral artery (median; $\mathrm{cm} / \mathrm{s}$; peak systolic $222 v 89, \mathrm{p}=0.002$; mean $169 v$ 57, $\mathrm{p}=0.001$ (Mann-Whitney). Pulsatility was significantly reduced in the feeder arter-

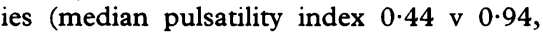
$p<0.001)$. Steal from the contralateral hemisphere was detected via the anterior communicating artery in six patients with parietal AVM. One brainstem AVM showed a steal from the anterior circulation via the posterior communicating arteries.

TCCS enables acquisition of real time haemodynamic data that may complement the anatomical information of standard neuroimaging techniques. Its safety and repeatability may be of benefit in the follow up of patients with AVM or in assessing the response to intervention.

EVALUATION OF ELIGIBILITY CRITERIA FOR RANDOMISED STUDY OF INTRACEREBRAL TUMOURS PRESENTING WITH EPILEPSY (MRC(BR08)) WHEN RETROSPECTIVELY APPLIED TO A BRAIN TUMOUR REGISTER J Miller, D Collie, PI Rothwell, J Ironside, R Grant. Western General Hospital, Edinburgh, UK

MRC(BR08) is a pragmatic randomised study of immediate $v$ delayed treatment for intracerebral tumours presenting with epilepsy, primarily designed for patients with low grade gliomas (LGGs). The SE Scotland Brain Tumour Register was analysed to determine the accuracy of the MRC(BR08) criteria and to see if postcontrast non-enhancement (PCNE) on CT increased specificity for LGGs.

MRC(BR08) identifies adult patients with CT evidence of a glioma who present with epilepsy without neurological signs or history of malignancy as being suitable for randomisation. Their criteria were applied to a database of incident cases of brain tumour diagnosed in 1989-90.

One hundred and five of 580 patients with intracranial brain tumours presented with epilepsy over a two year period. Twenty four $(23 \%)$ patients satisfied the criteria for randomisation for MRC(BR08): fourteen patients with LGG (10 histologically proved), nine high grade glioma (seven anaplastic astrocytoma, two glioblastoma multiformi), one undiagnosed tumour type. The inclusion of PCNE on CT identified 11 patients with LGGs (eight histologically proved) and one patient with anaplastic astrocytoma.

Thirty nine per cent of patients satisfying MRC(BR08) criteria had high grade gliomas. These would commonly be treated with early surgery and radiotherapy. The inclusion of PCNE on CT increased the specificity for LGGs.

INITIAL COGNITIVE CHANGES IN A PATIENT WITH FAMILIAL ALZHEIMER'S DISEASE

$S$ Newman, EK Warrington, A Kennedy, MN Rossor. National Hospital for Neurology and Neurosurgery, London, UK

Autosomal dominant diseases provide a unique opportunity to study the evolution of a pathological condition. The earliest clinical manifestations may be seen in longitudinal assessments of presymptomatic 
subjects "at risk". This methodology has been applied to investigate familial Alzheimer's disease.

A 43 year old woman "at risk" from a pedigree of necropsy-confirmed familial Alzheimer's disease was assessed on a comprehensive battery of neuropsychological tests in 1991 and subsequently these tests were repeated in early 1992 and late 1992 . These assessments included intelligence tests, and tests of memory, language, literacy skills, and perceptual functions. The selection of tests was determined by the availability of standardised scores so that performance across cognitive skills could be compared.

At the first and second assessment she was presymptomatic and at the second and third assessment her scores on the MMSE were still entirely normal (30/30 and 29/30). On neuropsychological assessment, however, a distinctive pattern of cognitive impairment was shown at the first assessment and progression of these deficits over the subsequent two assessments. These findings show that neuropsychometric methods have an important role in the early diagnosis and delineation of cognitive deficits in degenerative conditions.

IMMUNOSTAINING PATTERNS OF MONOCLONAL GM1 ANTIBODIES

G O'Hanlon, G Paterson, HJ Willison. University of Glasgow, UK

GM1 ganglioside IgG and IgM autoantibodies are associated with acute and chronic peripheral neuropathies respectively, including Guillain-Barré syndrome and multifocal motor neuropathy. A series of GM1 monoclonal antibodies have recently been cloned from the peripheral blood of patients with motor neuropathy. These antibodies all react with GM1 but differ in their precise fine specificities which are centred around the terminal $\mathrm{Gal}(, 1-3) \mathrm{GalNAc}$ epitope common to GM1 and the related glycolipids GA1 and GD1b.

These differences in fine specificity are reflected in the very variable patterns of immunoreactivity in different areas of the nervous system. Distinct patterns of staining in peripheral nerve myelin and nodes of Ranvier, have recently been seen, sites of interest as demyelinating conduction block is a feature of GM1 antibody associated neuropathy. Immunostaining may be localised in internodal myelin or may include the nodal region. Differences are analogous to the contrasting patterns of staining observed with the peanut lectin, arachis hypogaea and cholera toxin. Some GM1 antibodies show no preferential nodal staining.

These data demonstrate that GM1 antibodies are highly heterogeneous in terms of tissue reactivity, which may in turn be a reflection of their different immunopathogenic potential.

INDORAMIN FOR LOWER URINARY TRACT DYSFUNCTION IN MULTIPLE SCLEROSIS J O'Riordan, C Doherty, M Javed, D Brophy, M Hutchinson, D Quinlan. St Vincent's Hospital, Dublin, Eire

Lower urinary tract dysfunction is a major cause of morbidity in multiple sclerosis. a1 Adrenergic receptors are present in the bladder neck where increased adrenergic activity may contribute to diminished flow rates and urinary retention. Indoramin is selective $a 1$ adrenergic receptor antagonist. A randomised, placebo controlled study was performed to test the hypothesis that indoramin would improve bladder emptying in multiple sclerosis. Urinary function was assessed by measurement of peak and mean urinary flow rates, residual volume by ultrasound, and symptom score. The active treatment was $20 \mathrm{mg}$ indoramin twice daily. Eighteen men (of 41 assessed initially) were evaluated at entry to the trial and again after four weeks of treatment.

There was a mean percentage improvement in peak flow rate of $41 \%$ in the indoramin group compared with $-7 \cdot 4 \%$ in the placebo group $(p<0.05)$. There was no difference in residual urinary volumes between the two groups but patients taking indoramin reported a greater improvemen in urinary symptoms. Modulation of the $a 1$ receptor may have a part to play in the management of urinary dysfunction in multiple sclerosis.

LIMB ATAXIA, FACIAL NUMBNESS, AND ADIE'S PUPILS; ATAXIC GANGLIONOPATHY

II O'Riordan, M Javed, M Hutchinson. St Vincent's Hospital, Dublin, Eire

Eight women with features of an ataxic ganglionopathy with duration of illness from two to 20 years are described. The clinica features include an asymmetric sensory limb ataxia (five cases), generalised areflexia (eight), facial sensory loss (seven), and Adie's pupils, often subclinical (seven). The evolution was subacute or chronic with slow progression over many years; in two patients a diagnosis of central demyelination was initially considered. Four patients had evidence of a collagen disorder and one had a monoclonal gammopathy. Xerophthalmia was present in seven, and although Sjogren's syndrome was considered in all it was particularly difficult to prove. Motor conduction velocities were normal in all but in five women sensory nerve action potentials were absent or reduced.

Dorsal root ganglion biopsy in a few patients in other studies has shown evidence of a ganglionitis that also presumably affects the ciliary and gasserian ganglia; an autoimmune pathogenesis seems probable. The prognosis seems to be relatively benign. Immunosuppressive treatment has not been of use in management although plasma exchange has been helpful in the one woman with monoclonal gammopathy.

THE COST OF STROKE IN A DISTRICT HEALTH AUTHORITY: WHERE IS THE MONEY SPENT? S Pollock, S Wilock. Kent and Canterbury Hospital, UK

Current management of stroke is variable and in need of improvement. It is expensive, accounting for over $4 \%$ of the entire health budget nationally but as little is known about how the costs are distributed it is difficult to assess how money could be spent more effectively.

With information from the stroke admissions register, district paramedical and nursing activity analysis, acute unit management accounts, and extrapolated national statistics, the Canterbury and Thanet
Stroke Project has examined a bipolar district (population 305 000) without a stroke policy to estimate the 1991 expenditure on stroke and to define its component parts of prevention, acute care, and rehabilitation.

The estimated total cost of $£ 2859000$ represented $3 \cdot 1 \%$ of the annual budget, with nearly two thirds spent on chronic care and only a small fraction, $£ 77000$, mostly for anticoagulation, on prevention. Inpatient costs, spread equally between acute and non-acute beds, accounted for $60 \%$ of the budget. The mean duration of stay was 30 days of which 10 were spent in acute wards (cost $£ 161$ per day), and the remainder in non-acute beds, ( $£ 66$ per day) - that is, $\$ 3000$ per admission.

Nursing and infrastructure costs for both in and outpatients consumed $80 \%$ of the revenue, district nursing accounting for $£ 689000$. In sharp contrast only $£ 121000$, $4 \%$ of the budget, was spent on paramedical treatment.

Up to $60 \%$ of strokes are preventable and there is now good evidence that organised rehabilitation improves outcome. The findings presented here suggest that in the absence of a coherent strategy much of the current expenditure on stroke is inefficient and poor value for money.

THE EFFECT OF CYCLING ON H-REFLEX AMPLITUDE AND PRESYNAPTIC INHIBITION IN PARKINSON'S DISEASE

NJ Part, RC Roberts, TJ Ong, SV Wilson. University of Dundee, UK

The magnitude of the soleus H-reflex in normal subjects is greater at any given leve of muscle activity when the subject is performing static (for example, standing) as opposed to dynamic (for example walking, running, or cycling) activity. This is probably due to the activation of presynaptic inhibitory pathways acting on the soleus Ia afferent terminals. It has previously been shown that, at rest, transmission in presynaptic inhibitory pathways from anterior tibial muscle Ia afferents to soleus Ia afferent terminals is reduced in patients with Parkinson's disease compared with age matched controls. The effect of cycling on presynaptic inhibition in Parkinson's disease has now been investigated by determining the ratio of the gradients of the lines relating H-reflex amplitude to level of background EMG during static and dynamic soleus muscle activity. The average ratio (mean (SD)) for patients with Parkinson's disease was $1.32(\mathrm{SD} 0.24)(\mathrm{n}=7)$ and for age matched control subjects was 4.44 (SD $2 \cdot 33)(n=7)$. These values are significantly different ( $p<0.005, t$ test) and indicate impairment of activation of pathways mediating presynaptic inhibition of soleus Ia terminals during cycling in Parkinson's disease. The reduced levels of presynaptic inhibition allow increased transmission through the monosynaptic reflex pathway and may contribute to the increased muscle tone associated with the disease.

A PREVALENCE SURVEY OF MULTIPLE SCLEROSIS IN SUSSEX

M Rice-Oxley, ES Williams, JE Rees. Roya Sussex County Hospital Brighton, and Department of Public Health, Croydon, UK 
A first survey of the Brighton and MidDowns health districts in Sussex showed a prevalence of multiple sclerosis of $111 / 100000(95 \%$ CI 103-120) in a population of 596594 on prevalence day, 1 July 1991 . Cases were notified by multiple sources and to maximise case notification, patients were not contacted. Cases were classified by hospital and family practitioner notes. This is the first survey in the United Kingdom to use only the Poser criteria for classification. The Poser criteria are precise and exclude cases of isolated spinal cord syndrome. The Allison and Millar criteria used in the surveys in Scotland may lack specificity and it is concluded that the high prevalence of multiple sclerosis in Scotland needs to be reassessed with the more precise criteria. Until this is done, the latitude effect within the United Kingdom remains unproved.

SURVIVAL OF PATIENTS WITH IDIOPATHIC PARKINSON'S DISEASE

RAC Roos, J Jongen, EA van der Velde. Academic Hospital, Leiden, and State University, Leiden, The Netherlands

Variables that influence the progression and duration of illness of patients with idiopathic Parkinson's disease have been examined. This has been investigated by survival analysis with covariates employing the variables age at onset, initial symptom, age at reaching Hoehn and Yahr (HY) stage III, dementia free period, start of levodopa treatment, age at death, and expected age at death when the patient would not have had Parkinson's disease.

The files of 474 patients with parkinsonism who visited our department over the past 30 years were analysed, 345 (258 alive; 87 deceased) fulfilled the criteria of idiopathic Parkinson's disease. Men $(p=0.03)$ and patients with a tremor $(p=0.001)$ had a lower HY stage at the first visit in the Hospital. Probably tremor is more directly disabling and demanding of attention than hypokinesia. Patients with hypokinesia and rigidity progress more rapidly when the age at onset is between 60 and 80 years ( $p<$ 0.002 ). Probably hypokinesia is more sensitive in determining the HY stage. Patients with tremor become demented much later compared with the other group in the course of their disease $(60-80$ years; $\mathrm{p}<0.05$ ).

Overall no significant difference in survival between the first symptom type tremor $v$ hypokinesia was found. Neither the use nor the duration of levodopa influenced the duration of the disease, which is in discordance with most of the literature.

MUSICAL HALLUCINATIONS

JJ van der Sande, $M$ Shaya, HI Hamburger. Municipal Hospital Slotervaart, Amsterdam, The Netherlands

In the literature, musical hallucinations are reported to occur more commonly in females, and age, deafness, and brain disease of the non-dominant hemisphere may play a causal part.

Four patients with musical hallucinations are described. All were women, ranging in age from 68 to 85 years. Three patients were affected by a perceptive deafness, usu- ally mild. Lacunar infarctions in the right cerebral hemisphere were found on CT in two patients. One patient had undergone surgery for a right sided frontotempora meningioma 11 years previously. One patient did not show abnormalities on CT and MRI, but showed an irritative focal lesion deep in the right hemisphere on EEG with brain mapping.

It is concluded that dysfunction of the right cerebral hemisphere is probably more important than deafness in causing musical hallucinations.

A PRELIMINARY STUDY TO COMPARE FUNCTIONAL DISTURBANCE OF THE VISUAL PATHWAY WITH ANATOMICAL DEFICIT AS ASSESSED BY MAGNETIC RESONANCE IMAGING IN PAST DEMYELINATING OPTIC NEURITIS

R Sedhev, P Caruana, N Foster, N Haq, JR Heron, CP Hawkins. North Staffordshire Royal Infirmary, Stoke-on-Trent and Keele University, UK

Visual psychophysics is an established, sensitive method of detecting disturbance in magnocellular and parvocellular pathway function in demyelinating optic neuritis. The magnocellular route seems preferentially affected. MRI provides a means to assess the extent of optic nerve demyelination. Therefore magnocellular disturbance has been compared with lesions seen on MRI.

Six patients with past demyelinating optic neuritis and five healthy age and sex matched controls were studied. All had temporal modulation sensitivities at different temporal frequencies in the form of DeLange attenuation curves using three kinds of flickering stimuli with different spatial configurations. The mean threshold sensitivities for the control group were subtracted from the mean thresholds of the individual patients and the losses in the $\log$ modulation sensitivities were then calculated.

All patients had MRI of the optic nerves using STIR (2000/150/40) pre- and post-gadolinium DTPA. Slice thickness was $5 \mathrm{~mm}$. Optic nerve involvement was decided independently by $\mathrm{CPH} / \mathrm{NH}$ with good agreement. Lesion load varied from four $5 \mathrm{~mm}$ slices showing high signal to no abnormality seen in one patient.

In this small study no direct relation was found between functional and anatomical changes in past demyelinating optic neuritis (by visual psychophysics and MRI), nor between prolongation of VER and lesion load by MRI (correlation coefficient, $r=$ 0.35 ).

A larger study is required. However, two important questions are raised: firstly, whether past blood-brain barrier breakdown and inflammation rather than demyelination are responsible for magnocellular disturbance. Secondly, whether postchiasmal disease contributes significantly to sensory deficit.

CAROTID STENTING: AN ALTERNATIVE TO SURGERY FOR CAROTID PSEUDOANEURYSM? CR Sherrington, J Bamford, $M$ Nelson. St James's University Hospital, Leeds, and Leeds General Infirmary, UK

Pseudoaneurysm formation due to traumat- ic dissection of the internal carotid artery is uncommon. They may rupture, however, produce a mass effect, and be source of thrombosis causing recurren distal embolisation. Lesions may be inaccessible to surgery and carotid ligation may be required. This carries high morbidity.

A case of traumatic dissection of the extracranial internal carotid with pseudoaneurysm is presented, with recurrent thromboembolism despite anticoagulation. Aneurysm extension to the skull base made direct surgery not feasible, and therefore carotid ligation was considered. The absence of collateral flow to the ipsilateral hemisphere made the risk of both ligation and intravascular occlusion unacceptably high. A Stencker stent was therefore placed across the aneurysm neck via a transarterial approach. Follow up angiography two days and seven weeks after stenting showed increasing stasis in the sac and progressive isolation from the carotid lumen. Repeated transcranial doppler showed decreasing numbers of distal embolic signals over the same period. No further neurological events have occurred in the six month follow up.

It is concluded that carotid stenting is a feasible alternative technique to carotid ligation in inaccessible carotid pseudoaneurysm.

THALAMUS STIMULATION, A TREATMENT FOR PARKINSONIAN TREMOR

JD Speelman, DA Bosch. Academisch Ziekenhuis, University of Amsterdam, The Netherlands

Before the introduction of levodopa in the Netherlands in 1969 about 200 thalamotomies for tremor or rigidity in Parkinson's disease were performed annually, but only about 15 since then. In the past decade the number of patients referred for thalamotomy because of parkinsonian tremor and rigidity is increasing due to the complications of pharmacotherapy.

From the literature, it seemed that permanent relief of tremor and rigidity can be obtained for $80 \%$ of patients. In about $50 \%$ of the operations transient or mild side effects were found, with permanent severe complications in 4 to $9 \%$ especially after bilateral operation. Therefore, thalamotomy is only performed unilaterally, even in cases with severe, bilateral tremor.

The publication by Benabid in 1991 in The Lancet caused a renewed interest for permanent thalamic stimulation for the treatment of tremor resistant to pharmacotherapy, especially in Parkinson's disease. Thalamic stimulation is a surgical technique with placement of a permanent stimulation electrode stereotactically in the ventrolateral thalamus, connected with a subcutaneously placed stimulator, which can be switched on and off by the patient with a small magnet.

We consider that thalamic stimulation has the following advantages compared with thalamotomy: no or minimal structural brain tissue lesion; bilateral procedure possible; after unilateral coagulation a contralateral stimulation can be performed; less side effects.

Recently we have implanted three patients unilaterally. The short term follow up is favourable. 
INCREASED SPECIFICITY OF MAGNETIC RESONANCE IMAGING IN THE EARLY DIAGNOSIS OF MULTIPLE SCLEROSIS MW Tas, F Barkhof, MAA van Walderveen, OR Hommes, CH Polman, J Valk. Free University Hospital, Amsterdam and Institute of Neurology, Nijmegen, The Netherlands

Most studies show a high sensitivity of MRI in the diagnosis of multiple sclerosis, but a rather low specificity. An attempt to improve the specificity of MRI is reported.

Patients $(n=57)$ with monophasic neurological symptoms suggestive of multiple sclerosis were studied prospectively with MRI shortly after the onset of symptoms (mean interval five weeks). A spinal tap was performed in 34 patients. Clinically definite multiple sclerosis was diagnosed when new symptoms in other parts of the CNS occurred (mean follow up 13 months).

So far, multiple sclerosis developed in 17 patients $(35 \%)$. Other diagnoses were made in nine patients, and 31 patients remain without a diagnosis. Examination of CSF had a sensitivity of $69 \%$ and a specificity of $38 \%$. When four or more MRI lesions were required (Paty criteria), the sensitivity was $94 \%$ and specificity $55 \%$; with nine or more lesions these percentages were 88 and 75 respectively. Gadolinium enhancement increased specificity to $80 \%$, although sensitivity dropped to $55 \%$. The presence of an infratentorial lesion (Fazekas) had a sensitivity of $82 \%$ and a specificity of $80 \%$.

This prospective study shows that the specificity of MRI can be considerably increased by requiring either nine instead of four lesions, an enhancing lesion, or an infratentorial lesion.

AUTOSOMAL RECESSIVE HEREDITARY SENSORY NEUROPATHY WITH SPASTIC PARAPLEGIA

PK Thomas, S Wroe, P Misra, RHM King, K Bhatia, M Anderson, A Cabello, J Vilchez. Ipswich Hospital; Royal Free Hospital School of Medicine and Institute of Neurology, London; Midland Hospital for Neurosurgery and Neurology, Smethwick, UK; and Hospital La Fe, Valencia, Spain

A distinctive variety of hereditary sensory neuropathy is described in which a disorder of presumed autosomal recessive inheritance begins in childhood with a progressive distal sensory neuropathy affecting the lower limbs to a greater extent than the upper. This leads to a mutilating acropathy and is accompanied by a spastic paraplegia. Five patients with this syndrome have been investigated. These consisted of two sibling pairs with normal consanguinous parents and a sporadic case with normal unrelated parents. Spontaneous pain was a feature in two cases, which occurred in "crises" lasting several days in one. Nerve biopsies showed an axonopathy with loss of myelinated fibres of all diameters and loss of unmyelinated axons of lesser severity. There have been previous reports of a similar disorder with autosomal dominant inheritance but only a single report with possible autosomal recessive inheritance.
BRAIN AND SPINAL CORD MAGNETIC RESONANCE IMAGING IN MOTOR NEURON DISEASE

JW Thorpe, IF Moseley, CH Hawkes, WI McDonald, $\mathrm{DH}$ Miller. Institute of Neurology, London, UK

Pathological studies in motor neuron disease (MND) have on the whole shown more extensive changes within the spinal cord than the brain. Previous MRI reports have, however, concentrated on the brain findings. Spinal cord MRI and brain imaging in 11 patients with MND have been carried out. In all cases sagittal $\mathrm{T} 1$ and $\mathrm{T} 2$ weighted images of the cord were obtained, as well as T2 or $\mathrm{T} 22^{\star}$ weighted axial images through the cervical region. The sagittal images were unremarkable. In nine cases, however, axial imaging showed high signal within the anterolateral part of the cord. T2 weighted axial images of the brain showed a pathologically increased signal within the posterior limbs of the internal capsules in five patients, with variable extension rostrally to the corona radiata and caudally to the cerebral peduncles. Pronounced low signal within the motor cortex was seen in three patients. Only one patient had normal imaging of the brain and cord. It is concluded that spinal cord as well as brain MRI often shows characteristic abnormalities in MND, probably related to Wallerian degeneration within the pyramidal pathways MRI may be of considerable diagnostic value in patients with suspected MND.

SPINAL CORD MAGNETIC RESONANCF

IMAGING IN MULTIPLE SCLEROSIS: LESSONS FROM FAST STIR

JW Thorpe, BE Kendall, WI McDonald, DH Miller. Institute of Neurology, London, UK

Recent advances in the design of imaging sequences and receiver coils have greatly enhanced the ability of MRI to detect the spinal lesions of multiple sclerosis, while still relying predominantly on differences in transverse relaxation time (T2) to provide contrast. The short tau inversion recovery fast spin echo pulse sequence (fast STIR) combines the synergistic $\mathrm{T} 1$ and $\mathrm{T} 2$ contrast mechanism of conventional STIR with the reduced imaging time of fast spin echo (FSE). Fast STIR has been compared with T2 weighted FSE in evaluating the spinal cord lesions in 17 patients with multiple sclerosis, two patients with acute transverse myelitis, and 12 healthy controls. Intrinsic lesions were seen only in patients. Twenty five of the 62 lesions identified were more clearly shown by fast STIR, 23 by FSE. Signal to noise and contrast to noise ratios were higher, however, in all cases on FSE, suggesting that other mechanisms must play a part in lesion conspicuity, including the brightness of surrounding structures and the choice of window settings. Such factors should be considered in comparative studies of this kind.

\section{WRONG-WAY EYES}

CC Tijssen. St Elisabeth and Maria Hospitals, Tilburg, The Netherlands

Conjugate eye deviation (CED) in patients with supratentorial stroke is generally directed ipsilateral to the lesioned hemisphere. Incidentally, CED occurs to the contralateral side, also designated as wrongway eyes. Five new cases are reported and previously published reports are reviewed to elucidate the lesion responsible and the mechanism underlying this phenomenon.

In a prospective study of 133 consecutive patients with CED caused by an acute supratentorial stroke, five patients had contralateral CED. These five patients were studied clinically; in four patients CT was performed. Four of the five patients died and necropsy was performed in all four of them.

In four patients contralateral CED was caused by an intracerebral haemorrhage located thalamic $(n=2)$, frontoparietal ( $n=$ $1)$, and frontoparietotemporal $(n=1)$. In one the cause was a subdural haematoma. All patients had clinical signs of rostral midbrain dysfunction and a shift of midline structures on CT or at necropsy. Necropsy showed no additional destructive lesions in the other hemisphere or brainstem, except for one patient who showed a demyelination of the medial part of the cerebral peduncle ipsilateral to the thalamic haemorrhage at midbrain level, at microscopical examination.

It is concluded that contralateral CED is always associated with haemorrhagic lesions, most commonly in the thalamus The prognosis of patients with this sign is generally poor. Involvement of descending oculomotor pathways from the contralatera hemisphere at the midbrain level is the most probable explanation for the phenomenon.

DISTAL MYOPATHY IN A DUTCH FAMILY: A VARIANT OF WELANDER MYOPATHY?

$M$ de Visser, JWHJ Dammers. Academic Medical Center, Amsterdam and Medical Center, Alkmaar, The Netherlands

Welander myopathy is an autosomal dominantly inherited distal myopathy most often found in Sweden. The clinical picture is characterised by slowly progressive weakness of the extensors of hands and fingers with onset usually in the fifth decade. EMG and muscle biopsy show predominantly myopathic changes, although a neurogenic component may be present even at an early stage of the disease. Rimmed vacuoles are found in a large proportion of the muscle biopsies. Non-Swedish families with distal myopathy of the arms are rare.

A Dutch family with early onset distal myopathy starting in the hands is described. Five affected persons from a Dutch family were clinically examined. In two out of five electrophysiological examination was carried out and a muscle biopsy specimen was taken. A sural nerve biopsy was performed in one of these two patients. Clinical examination showed asymmetric weakness predominantly affecting the finger extensors. The proximal muscles and the lower extremities were not affected. Sensation was normal and myotactic reflexes were preserved. EMG showed both polyphasic motor unit action potentials (MUAPs) of short duration, and fast recruitment, and polyphasic MUAPs of long duration. Motor and sensory nerve conduction velocities were normal. Histopathological examination of muscle also disclosed myopathic and neurogenic abnormalities. In one muscle biopsy an extensive endomysial mononuclear cell 
infiltrate was seen. Rimmed vacuoles were absent. At the ultrastructural level, no filamentous inclusions were detected. The nerve biopsy showed an increased variation in the size of large fibres and signs of remyelination. It is concluded that this family has a disorder which primarily affects the extensors of the fingers and resembles Welander's myopathy as regards distribution of weakness and the presence of myogenic and neurogenic changes on EMG and in muscle biopsy. Atypical features consist of non-Swedish descent, an onset in early adulthood, and the absence of rimmed vacuoles.

OBSTETRIC BRACHIAL PLEXUS LESION: ONLY A PERIPHERAL LESION?

JW Vredeveld, CAM Rozeman, ACJ Slooff. Ziekenhuis De Wever and Gregorius, Heerlen, The Netherlands

Apart from adults and adolescents with brachial plexus lesions, in this hospital more than 250 children were seen with an obstetric brachial plexus lesion and still severe paresis after several months. As about $90 \%-95 \%$ recover within a short time, this group represents a total cohort of about 2500-5000 neonates with an obstetric brachial plexus lesion.

In adults and adolescents, the EMG findings closely mimic the clinical picture and give reliable information about the severity and location of the lesion. In neonates, the EMG findings are different and mostly much more "optimistic" than the clinical lesion.

One of the most striking differences is the finding of a mixed interference pattern in the EMG in a muscle that is clinically completely paralysed. Sometimes painful stimuli are needed for this finding. This was found in more than half of the children. Therefore, the paralysis in these children cannot be explained by the peripheral lesion alone. The same was found by McComas in one child, using his motor-unit counting technique.

This leads to the conclusion that changes in the CNS, either induced by or resulting from the lesion in the peripheral nervous system, may play an important part in children with an obstetric brachial plexus lesion.

CORRELATION OF QUANTITATIVE MAGNETIC RESONANCE IMAGING ABNORMALITIES AND DISABILITY IN MULTIPLE SCLEROSIS MAA van Walderveen, F Barkhof, OR Hommes, CH Polman, J Valk. Free
University Hospital, Amsterdam and Institute of Neurology, Nijmegen, The Netherlands

MRI is often used as an outcome measure in phase II trials in multiple sclerosis, assuming that MRI activity predicts clinical disability. The relation between increase in MRI lesion load and clinical disability has been studied.

Patients with multiple sclerosis $(n=48)$ were examined twice clinically (EDSS) and with MRI (mean interval 24 (range 10-42) months). MRI (spin echo sequences) included T2 weighted images in all and T1 weighted images in 18 patients, which were computer quantified with a seed growing technique. An increase in EDSS correlated with increase in T2 weighted lesion load $(r=0.53, \mathrm{p}<0.0001)$, whereas the subgroup with $\mathrm{T} 1$ weighted images showed a significant correlation of increase in EDSS and area of decreased signal intensity $(r=$ $0.89, \mathrm{p}<0.0001$ ).

It is concluded that short duration of follow up might explain the weak relation between increase in disability and T2 lesion load (25\% variance explained). More probably T2 weighted images also detect areas of oedema and mild demyelination, which are still functionally intact, whereas areas of decreased signal intensity on $\mathrm{T} 1$ weighted images represent only severe demyelination, pronounced gliosis, and axonal loss, having little residual function; hence the strong correlation with increase in EDSS $(80 \%$ variance explained).

THE NEUROPHARMACOKINETICS OF ACUTELY ADMINISTERED PHENYTOIN IN THE RAT

MC Walker, MS Alavijeh, PN Patsalos, SD Shorvon. Institute of Neurology, London, UK

Phenytoin is given acutely in the treatment of status epilepticus. Although the plasma pharmacokinetics of phenytoin have been well described, the neuropharmacokinetics of phenytoin in brain extracellular fluid (a pharmacodynamically relevant compartment) have not been determined.

With a newly developed animal model that allows concurrent sampling of blood and brain extracellular fluid by stereotactically implanted microdialysis probes, the kinetics of phenytoin ( $50 \mathrm{mg} \mathrm{kg} \mathrm{g}^{-1}$ ) in blood, frontal cortex, and hippocampus have been compared in Sprague-Dawley rats $(n=7)$.

Phenytoin appeared rapidly in the brain compartment; however, hippocampal phenytoin exhibited a double peak ( $T_{\max } 25$ and 60 minutes) by contrast with the frontal cortex which peaked ( $T_{\max } 15$ minutes) and then plateaued. Concentrations then fell exponentially in both brain areas with a half life similar to that in plasma (70(SD 17) minutes). Area under the curve values in the hippocampus (521(SD 34) umol min $1^{-1}$.... were significantly $(\mathrm{p}<0.05)$ higher than that in the frontal cortex (326 (SD 50) $\mu \mathrm{mol}$. min. $1^{-1}$ ) This study emphasises the complex nature of brain neuropharmacokinetics and the fact that plasma and total brain drug concentrations may be poor indices of brain extracellular fluid concentrations. The relevance of this in the treatment of human status epilepticus will be discussed.

OBSERVER VARIATION IN REPORTING

CONVENTIONAL AND MAGNETIC RESONANCE ANGIOGRAMS OF THE CAROTID BIFURCATION GR Young, PRD Humphrey, MDM Shaw, TE Nixon, ETS Smith. The Walton Centre for Neurology and Neurosurgery, Liverpool, UK

Magnetic resonance angiograms (MRAs) and digital subtraction angiograms (DSAs), of the internal carotid artery bifurcations of 70 patients (137 vessels), were reported by two experienced neuroradiologists. Each angiogram was assessed blind and independently, by recording the visual impression of the degree of stenosis. One radiologist reported all the angiograms a second time, enabling full analysis of the inter and intraobserver variation in reporting. A separate analysis was performed with calipers by one of the authors (GY), who measured the minimum residual lumen of the internal carotid artery as numerator and the maximum diameter of the distal common carotid artery as denominator, these being hard, objective measurements.

For interobserver variation, the means of the absolute differences between assessments were $7.0 \%$ for DSA and $6.4 \%$ for MRA. Corresponding results for intraobserver variation were $5 \cdot 7 \%$ and $7 \cdot 7 \%$. Intraobserver mean absolute differences with caliper measurements were $3.0 \%$ for DSA and $3.5 \%$ for MRA. Clinically important differences occurred in $5.5 \%$ (DSA) and $4 \cdot 1 \%$ (MRA), due to interobserver variation, and in $5.3 \%$ (DSA) and $5.3 \%$ (MRA) because of intraobserver variation. Intraobserver variation in caliper readings resulted in clinically important differences in $3.1 \%$ (DSA) and $1.6 \%$ (MRA).

Observer variation in reporting MRA is similar to that for DSA, and can result in clinically significant differences. Caliper measurements would seem to be more consistent. 